\title{
ENGINEERING OPERANDO METHODOLOGY: UNDERSTANDING CATALYSIS IN TIME AND SPACE
}

Raquel Portela, Susana Perez-Ferreras, Ana Serrano-Lotina, Miguel A. Bañares* Instituto de Catálisis y Petroleoquímica, ICP-CSIC, Marie Curie 2, Madrid, Spain

\section{Content}

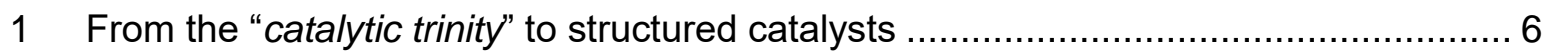

2 Shedding light into the "black box": the operando methodology ............................ 8

3 Chemical structure and activity in the operando methodology.............................. 10

3.1 Catalytically relevant chemical structures .............................................. 10

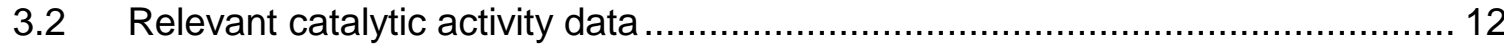

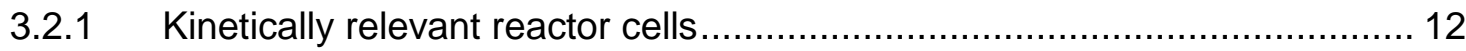

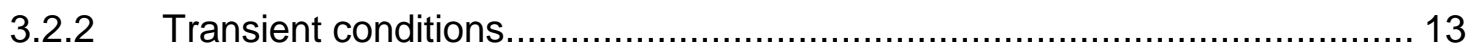

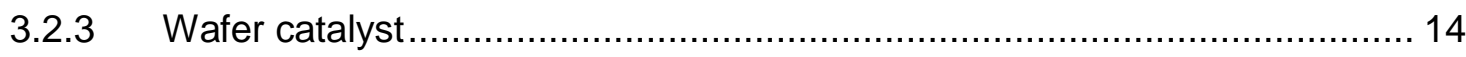

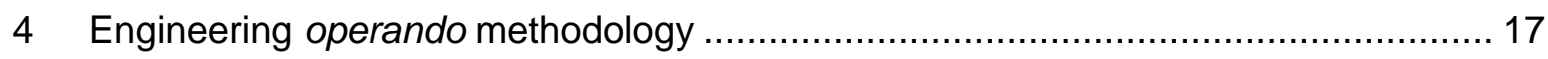

4.1 From powders towards shaped bodies .............................................. 17

4.2 Local operando studies in integral reactors................................................ 18

4.3 Space-resolved operando studies........................................................ 20

4.3.1 Mapping solids during operando studies ........................................... 20

4.3.2 Mapping the fluid phase composition and states during operation ..............26

4.3.3 Temperature mapping in operando studies...................................... 30

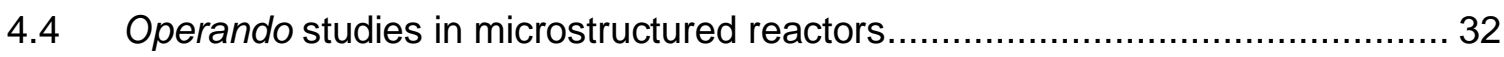

5 Concluding Outlook: measure locally, think globally....................................... 36

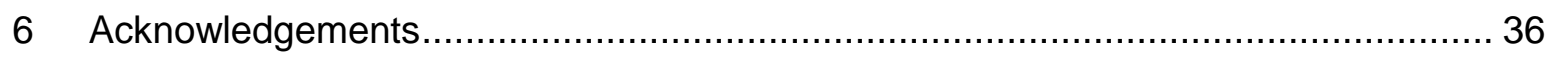

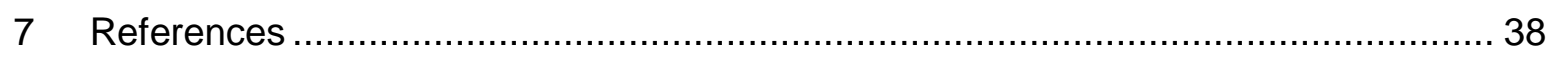




\section{Figures}

Figure 1. Multiparametric evolution in catalyst development process. Reproduced with permission from [1].

Figure 2. Overview of the spatial (left) and temporal (right) resolution of vibrational microspectroscopic techniques applied in heterogeneous catalysis. Adapted from [50]....

Figure 3. Operando Raman-GC study of a $\mathrm{VOx} / \mathrm{Al}_{2} \mathrm{O}_{3}$ catalyst. Raman spectra at different reaction temperatures (left) and activity data vs. reaction temperature (right) during (TOP) propane $\mathrm{ODH}$, and (BOTTOM) propane $\mathrm{DH}$ and in situ reoxidation at $773 \mathrm{~K}$. Catalyst weight: $150 \mathrm{mg}$, total flow: $67 \mathrm{ml} \cdot \mathrm{min}^{-1}$, reaction feed: propane/oxygen/helium=1/6/4 for $\mathrm{ODH}$ and 1/0/6 for $\mathrm{DH}$. Reproduced with permission from [68]. 11

Figure 4. Arrhenius plots obtained in an operando fixed-bed reactor using Raman spectroscopy with gas cromatography during ethane oxidative dehydrogenation on vanadium oxide molecularly dispersed on ceria. Reproduced with permission $[68,70] \ldots 12$ Figure 5. Relative evolution of the FTIR bands of ${ }^{12} \mathrm{C}$-containing carbonate, ${ }^{12} \mathrm{C}$-containing formate and ${ }^{13} \mathrm{CO}_{2}$ at $155{ }^{\circ} \mathrm{C}(\mathrm{A})$ and $220{ }^{\circ} \mathrm{C}(\mathrm{B})$ under $2 \%{ }^{13} \mathrm{CO}+7 \% \mathrm{H}_{2} \mathrm{O}$, following steadystate under $2 \%{ }^{12} \mathrm{CO}+7 \% \mathrm{H}_{2} \mathrm{O}$. Reprinted with permission from [75] ......................... 14 Figure 6. Sintering mechanism on Pt/ZSM-5 catalysts on the external surface and in the mesopores according to 2D IR pressure-jump spectroscopy of adsorbed CO. Reproduced with permission from [76]. 14

Figure 7. Left: 'Sandwich' IR reactor cell (A) longitudinal, and (B) radial views. 1 Thermocouple location, 2 - KBr windows, 3 - O-ring, 4 - Gas inlet, 5 - Sample, 6 Sample holder, 7 - Gas outlet. (C) Circular, and (D) square sample holders. Right: Flow model in the round sample holder (left panel) and evolution of the concentration with time (right panel). Reprinted with permission [59] 15

Figure 8. Pore size distribution (line) and cumulative volume (dotted line) for uncompacted and compacted titania-supported vanadia-tungsta catalyst at different pressures [81].... 16 Figure 9. Effect of pelletizing pressure and wafer thickness on catalyst efficiency and intrinsic activity during NOx selective catalytic reduction on titania-supported vanadiatungsta wafers in an operando transmission IR reactor. Reproduced with permission from [81]. 17

Figure 10. Vibrational spectroscopy operando reaction cells for monoliths. Left: transmission FT-IR, reproduced with permission from [39]. Right: Raman, reproduced with permission from [15]. 18

Figure 11. Transient state operando study on a $\mathrm{V}_{2} \mathrm{O}_{5}-\mathrm{WO}_{3} / \mathrm{TiO}_{2}-$ sepiolite wafer catalyst. TOP: FTIR spectra contour plot showing the growth of ammonia-derived species on the surface after changing from $20 \% \mathrm{O}_{2} / \mathrm{Ar}$ to SCR conditions. MIDDLE: FTIR area profiles of $\mathrm{NH}_{3, \text { ads }}$ and $\mathrm{NH}_{4}{ }^{+}$. BOTTOM: evolution of gas phase species concentration measured by MS. Reproduced with permission [90]. 19

Figure 12. (TOP), schematic illustration of a cell for space- and time-resolved DRIFTSRaman experiments. (BOTTOM), DRIFT (A-C) and Raman (D-F) spectra during NOx storage reduction (NSR) at the front, middle, and back positions of a Pt$\mathrm{Ba} / \mathrm{CeO}_{2}$ catalyst bed [18]. 21

Figure 13. Methanation over $\mathrm{Ni} / \mathrm{Y}-\mathrm{Al}_{2} \mathrm{O}_{3}$ monolithic catalyst. The activity was followed by MS. The stability of crystalline and non-crystalline distributions was proven by $\mu$-XRD-CT 
(2D during heat ramping, then $3 \mathrm{D}$ ) and $\mu$-absorption-CT. The high quality summed $1 \mathrm{D}$ XRD patterns confirmed that no reaction intermediates were formed. Figures reproduced with permission from [102]. 23

Figure 14. Reconstructed 2D weight percent composition maps of $\mathrm{Co} / \mathrm{y}-\mathrm{Al}_{2} \mathrm{O}_{3}$ during $\mathrm{H}_{2}$ reduction and Fischer-Tropsch synthesis and composition profiles compiled from the integrated and scaled reflection intensities for the various cobalt-containing phases from the summed 2D diffraction data. Adapted from [103]. Figure cited with permission of ACS as source. https://pubs.acs.org/doi/full/10.1021/acscatal.6b03145. Further permissions related to the material excerpted should be directed to the ACS 24

Figure 15. X-ray radiography images showing the evolution of surplus electrolyte ring between the separator and the lithium anode hole at different states $(A-H)$ of charge/discharge (\%) during the first cycle. The plot shows the charge/discharge curve and the measured thickness $d$ (red color in the scheme and the plot). Reproduced with permission from [107]. 25

Figure 16. Calculated velocity profile in a monolith showing the negligible effect of a capillary MS probe situated in the corner of the central channel. $\mathrm{T}=200^{\circ} \mathrm{C}, \mathrm{u}_{0}=0.016 \mathrm{~m} \mathrm{~s}^{-1}$, channel size $=0.001 \mathrm{~m}$, probe diameter $=250 \mu \mathrm{m}$. Reproduced with permission from [111].

Figure 17. Simultaneous measurement of thermal, kinetic and spectroscopic profiles through a fixed-bed tubular reactor. Left: Catalytic bed of $50 \mathrm{wt} . \% \mathrm{MoO}_{3} / \mathrm{Y}$-alumina spheres, middle: probe geometry, right: Raman spectra, temperature and composition profiles. Reproduced with permission from [117]. 27

Figure 18. 2-D slice section through 3-D MR images of water distribution within an initially water-saturated packing of 500- $\mu \mathrm{m}$ glass spheres. Voxel resolution is $94 \mathrm{~mm} \times 94 \mathrm{~mm} \times$ $94 \mathrm{~mm}$. Data are shown before drying commenced and at three time intervals during the drying process. Only the water within the inter-particle space of the bead packing was imaged (white pixels). No signal was obtained from the solid and gas phases present. Reproduced with permission from [123, 124]. 28

Figure 19. ${ }^{1} \mathrm{H}$ zero-time echo images and axial slices extracted from the corresponding 3D dataset obtained from a membrane-electrode assembly (MEA) operating with $\mathrm{H}_{2}$ and air at $80 \stackrel{\circ}{\circ}$ and $30 \mathrm{~mA}$. (a) Fuel cell at $25 \stackrel{\circ}{\circ} \mathrm{C}$ before operation ( $0 \mathrm{~V})$; (b) $\mathrm{t}=0(0.44 \mathrm{~V})$; (c) $\mathrm{t}=$ $420 \mathrm{~min}(0.42 \mathrm{~V})$; (d) fuel cell at $25{ }^{\circ} \mathrm{C} 14$ hours after the switch off at $420 \mathrm{~min}$. Reproduced with permission from [125]. 28 Figure 20. 2-D MR image of an oscillating chemical reaction occurring within a bed packed with glass beads. Chemical waves are imaged as a result of the oscillatory production of $\mathrm{Mn}^{2+}$ and $\mathrm{Mn}^{3+}$ species, identified as dark and light bands, respectively. Reproduced with permission from [126]. 29

Figure 21. Operando study of ethylene hydrogenation over cordierite monoliths coated with $1 \%$ wt. $\mathrm{Pt} / \mathrm{Al}_{2} \mathrm{O}_{3}$ (a) Cross-section photographic image of the honeycomb catalyst, (b) NMR ethylene image of the cross section under a non-reactive mixture of ethylene and argon measured by 3D MRSI, (c) and (d) ethane concentration (\%vol) maps at low and high flow rate, respectively. e) Experimental and model ethane/ethylene ratio profile of the low flow-rate experiment. Adapted from [127]. 30 
Figure 22. 2D ${ }^{1} \mathrm{H} M R I$ data from which bed porosity, liquid holdup and wetting efficiency of $0.3 \mathrm{wt} \% \mathrm{Pd} / \mathrm{Al}_{2} \mathrm{O}_{3}$ catalyst pellets were calculated. (a) bed flooded with liquid 1-octene, and (b) bed during 1-octene hydrogenation reaction. Reproduced with permission from [129].

Figure 23. Thermographs showing an ignition sequence on a $5 \mathrm{wt}$. \% $\mathrm{Rh} / \mathrm{SiO}_{2}$ catalyst wafer; the flow is parallel to the wafer surface, flowing from the bottom left corner to the top right one. Reproduced with permission [132, 133].

Figure 24. Space-resolved operando study on the ignition of the catalytic partial oxidation of methane in a fixed-bed capillary microreactor. (a)-(d) Oxidized catalyst (yellow-orange), formation of the front of reduction (red-violet), advance of the front towards the inlet. (e) Xray absorption image where the single reduced particles can be identified (f) Temperature profile evolution measured by IR-thermography. Reproduced with permission from [136].

Figure 25. Operando Raman-GC results of silver catalyst during oxidation/reaction cycles at $773 \mathrm{~K}$. Raman spectra at the end of: (a, c, e) oxidation, and (b, d, f) reaction steps. Oxidation in a flow of $\mathrm{O}_{2}(4.1 \%)$, $\mathrm{He}$ as balance; reaction in a flow of $\mathrm{CH}_{3} \mathrm{OH}(8.75 \%), \mathrm{O}_{2}$ (3.5\%) and $\mathrm{H}_{2} \mathrm{O}(6.63 \%)$, He as balance. Reproduced with permission from [137]......... 33 Figure 26. Space- and time-resolved data during $\mathrm{CO}$ oxidation at $110^{\circ} \mathrm{C}$ with a Pt catalyst in a capillary reactor in $1000 \mathrm{ppm} \mathrm{CO}, 10 \% \mathrm{O}_{2}$ in $\mathrm{He}$, total flow of $50 \mathrm{~mL} \cdot \mathrm{min}^{-1}$. (a) Catalyst bed scheme with the characterization points location and global gas-phase MS results. (b) LCF of XANES spectra. (c) k2-weighted Fourier-transformed QEXAFS data (k-range: 3.0$9.0 \AA^{-1}$ ). Reproduced with permission form [138]..... 34 Figure 27. Map of a $\mathrm{Rh} / \mathrm{Al}_{2} \mathrm{O}_{3}$ catalyst during methane partial oxidation. (a) Oxidized Rhspecies; (b) reduced Rh-species; (c) featureless background; (d) relative concentration of the oxidized and reduced Rh-particles in the axis of the fixed-bed (conditions: $\mathrm{T}=362{ }^{\circ} \mathrm{C}$, space velocity $\left.=1.9 \times 10^{5} \mathrm{~h}^{-1}\right)$. Reproduced with permission from [139]. 35 Figure 28. 3D plots of Raman intensity in the T-junction region for specific bands: (a) 893 $\mathrm{cm}^{-1}$ from acetic acid, and (b) $882 \mathrm{~cm}^{-1}$ from ethanol. Reproduced with permission from [141]. 35 


\section{ABSTRACT}

The term operando was coined at the beginning of this century to gather the growing efforts devoted to establish structure-activity relationships by simultaneously characterizing a catalyst performance and the relevant surface chemistry during genuine catalytic operation. This approach is now widespread and consolidated; it has become an increasingly complex but efficient junction where spectroscopy, materials science, catalysis and engineering meet. While for some characterization techniques kinetically relevant reactor cells with good resolution are recently developing, the knowledge gained with magnetic resonance and X-ray and vibrational spectroscopies studies is already huge. The scope of operando methodology with these techniques is recently expanding from studies with small amounts of powdered/pelletized solids to more real catalytic systems. Engineering catalysis implies larger physical domains, and thus all sort of gradients. Space- and time- resolved multi-technique characterization of both the solid and fluid phases involved in heterogeneous catalytic reactions (including temperature data) is key to map processes with different perspectives: taking into account existing heterogeneities at different scales, and facing up- and down-scaling, for applications ranging from microstructured reactors to industrial-like macroreactors (operating with shaped catalytic bodies and/or in integral regime). This work reviews how operando methodology is evolving towards engineered reaction systems. 


\section{From the "catalytic trinity" to structured catalysts}

The nature of the catalytic act is the same for any kind of catalysis, be it homogeneous, enzymatic or heterogeneous; to some extent, we may take the academic liberty to call these the "catalytic trinity", in the sense that the three are one. Catalysis is a phenomenon that occurs at the atomic scale when the molecules of reactants interact with active sites; such interaction weakens bonds, rearranges them, and electrons and atoms dance into new molecules. However, while the catalytic act takes place at the atomic scale, applied catalysis is framed in larger scale constraints. In the case of homogeneous and enzymatic catalysis it is critical to ensure that the reaction medium is free of diffusional limitations. Heterogeneous and enzymatic catalysis encompass additional hurdles in the near field: molecules mobility to reach and depart from the catalytic site is critical to avoid diffusional control. Large scale catalytic systems bring additional difficulties to enable an atomic scale phenomenon to occur within the meters range. Gradients become ubiquitous, and they have to be known and steered to optimize catalytic performance.

In this review, we are going to focus on heterogeneous catalysts, which can be organometallic compounds, immobilized enzymes, or metals or metal oxides bond to silicates, carbonaceous substrates, oxides or zeotypes, among others. More precisely, we will focus on how characterization should engage the process of bringing a catalytic system idea into a real working catalytic reactor, which is a multistage enterprise that progressively converts a model/research seminal concept into a technical/industrial final process, as Figure 1, from a recent review [1], nicely reflects.

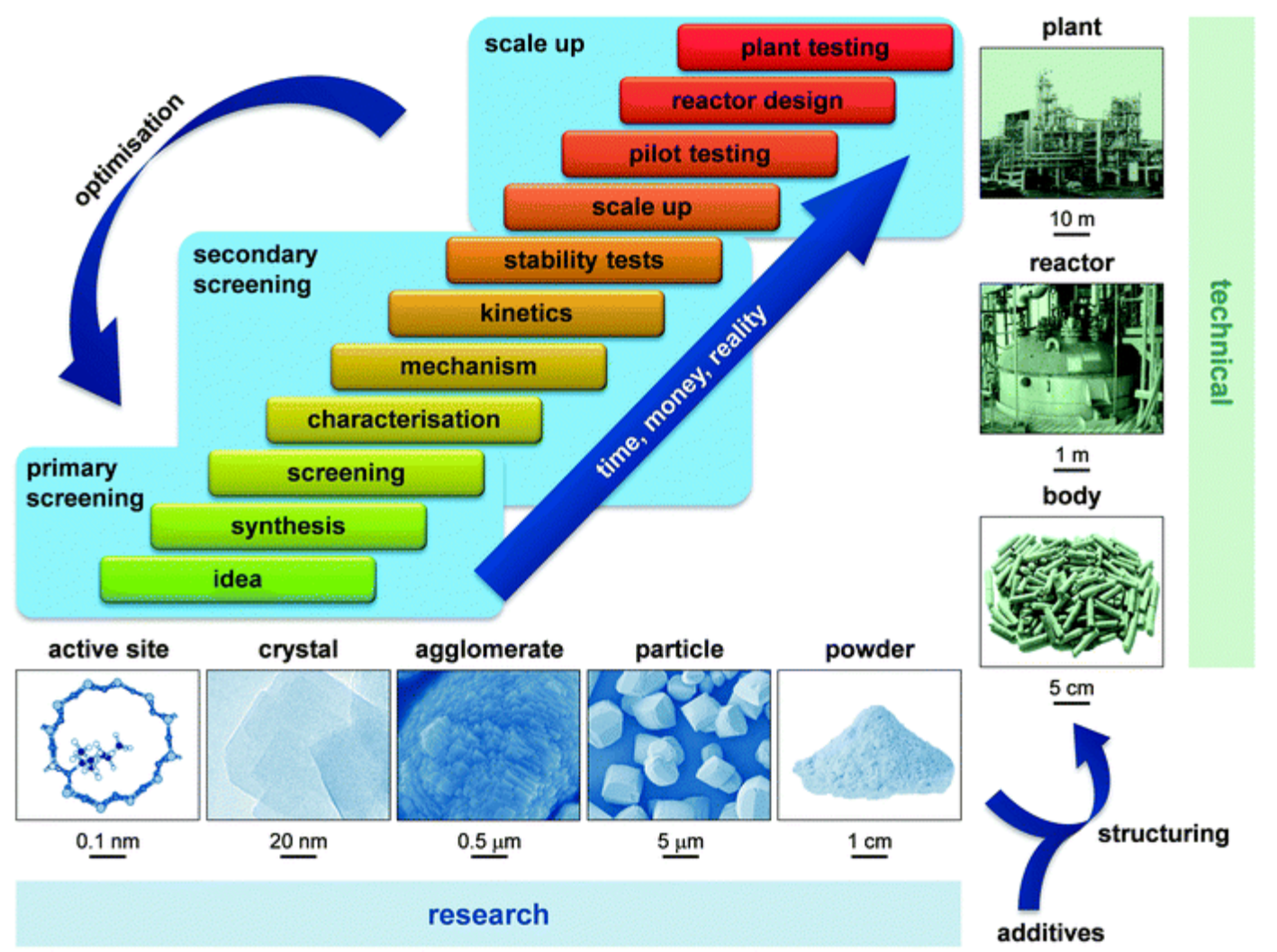

Figure 1. Multiparametric evolution in catalyst development process. Reproduced with permission from [1]. 
Chemical engineering provides a phenomenological approach to optimize the performance of large reactors, combining reaction modeling with reactor design. Cellular or structured catalytic reactors are gaining interest over conventional packed bed reactors due to the low pressure drop, high geometric specific area, low axial dispersion and back-mixing, reduced fouling or plugging, and easy cleaning and scale-up [2-4]. Honeycomb monoliths are increasingly investigated for a wide range of reactions in the environmental remediation field and the chemical/petrochemical/biochemical industries [5]. Similarly, in the past two decades also foams have deserved attention in the catalytic field [6]. Thus, shaping/structuring becomes a key stage in the manufacturing of catalysts, because it determines the final geometry (fluid dynamic properties) and composition (physicochemical properties), and therefore the performance of the catalytic body; characterization cannot be performed obviating this step.

Another approach to engineer catalysis is going small, taking advantage of microstructured reactors. These are reactors with three-dimensional structure and inner dimensions between 10 and $100 \mu \mathrm{m}$; their main feature, in comparison to macroreactors, is the high surface-to-volume ratio [7]. Microreaction technology is an interdisciplinary field that has gained significant importance due to the numerous advantages with respect to conventional systems, such as the wider operating window and tighter process control provided by the enhanced rate of heat and mass transfer [8]. The former capability, for example, allows fast heating and cooling, suppression of hot spots and a safely operation in processes which would be within the explosive regime in conventional reactors [9, 10]. The latter permits, among others, higher selectivity of the target product by preventing undesired consecutive or side reactions [11]. The transport intensification inherent to microstructured reactors makes them useful as laboratory tools so that even fast, highly exothermic reactions can be performed isothermally and in the absence of mass transfer limitations. Furthermore, safer operation due to the small channel dimensions allows exploration of new reaction pathways, in addition to process intensification through high pressure and temperature chemistries [12]. Heterogeneous catalytic microreactors are based mostly on reactive membranes, inert membranes, or non-porous microchannels with packed beds or wall-coated catalysts [13].

Little research is devoted to understanding the additional complexity of engineered, industrially relevant catalytic systems. Moreover, structured and packed bed (micro)reactors may be highly heterogeneous due to non-uniform distribution of active phase or reactants, or to non-uniform reaction progress within shaped catalysts. Consequently, advanced analytical tools [14] and adapted reaction test rigs [15] are needed not only to probe the composition and structure on nano- to millimeter-length scales, but also their effect on the final performance of the developed catalyst.

The operando methodology provides a direct grip between the working catalyst site structure and its performance. It has been evidenced as an invaluable tool to understand catalysis [16-32] and as method for real-time operation control [33]. The value of the operando methodology is that it characterizes a working catalyst and simultaneously measures its performance. For this reason, the operando reactor-cell has to be designed to perform like the corresponding real system, delivering reliable catalytic data. There is a growing awareness of the need to engineer operando methodology in order to be able to map working catalysts and catalytic reactions within reactors in the final shape with enough time- and space-resolution to understand and optimize catalytic processes [15, 29, 34, 35]. This is poised to deliver two major outcomes: a fundamental and an applied one. 
On the one hand, we will be able to understand chemical structure-activity relationships in a holistic manner, with resolution in time and space. On the other hand, it will provide key knowledge for real-time monitoring of the catalyst and the catalytic reaction, endowing us with the ability to implement solid Process Analytical Technology systems (PAT) [36].

\section{Shedding light into the "black box": the operando" methodology}

In the past decades, the huge advance in molecular calculations and characterization technologies has pushed the progressive substitution of the empirical trial-and-error research approach by a rational molecularly driven design of active sites to optimize their quality, strength, concentration, distribution and accessibility [22]. The "black box" approach, where catalysts are characterized before and after reaction, fails to provide complete information of materials microstructure, composition and reactivity because these may be sensitive to the temperature, pressure, irradiation, and composition of the reactive environment. The "materials gap", the "temperature gap" and the "pressure gap"[23] have been nowadays mostly overcome with the development of in-situ techniques [26]. The operando methodology has gone a step forward, combining the characterization of a catalytic material during reaction (in situ characterization) with the quantitative assessment of the actual performance of the catalyst, i.e. with the simultaneous analysis of the fluid stream, to calculate, for instance, conversion and selectivity. Detailed physicochemical characterization of the solid and fluid phases during operation in realistic conditions helps to understand: i) the mechanisms of the adsorption/desorption, transport, and reaction processes taking place and ii) the behavior of precursors, active and adsorption sites, reactants, intermediates, products, spectators, and poisonous species. This insight stands on spectroscopy, which in turn stands on the interaction of different electromagnetic radiation with matter. Virtually, catalysis cannot be understood without spectroscopy, which provides multiple complementary insights into the state of matter.

Spectroscopy has been profitably used with the operando approach to determine the nature, quantity, structure and environment of atoms, ions and molecules [30]. Among them, UV-vis is a powerful method to probe the electronic transitions of organic deposits [37] and $d-d$ transitions of transition metals [34]; as a whole, it delivers information on the oxidation state, chemical environment and domain sizes [38]. Molecular spectroscopies, like Raman and infrared, deliver complementary information on local molecular structures based on their vibrational states [22, 30, 35, 39]. Raman is a two-photon phenomenon more sensitive to covalent bonds vibrations, where polarizability changes, while infrared is a single-photon absorption phenomenon, more sensitive to ionic bond vibrations, where dipolar moment changes [40]. Different selection rules endow Raman and infrared with different and complementary sensitivities; e.g., for heterogeneous catalysts, Raman is preferentially used to characterize catalyst chemical structure at work and, to a lesser extent, to monitor adsorbed molecules, while infrared has the inverse distribution of applications. But both are sensitive to the states of the catalyst, adsorbed reactants and, depending on the system, to the reactants/products prior/after interaction with the active site.

1 CLARIFICATION: The term should be "operando" and not "in operando". Unlike "in situ", "operando" may not be preceded by "in". While "In situ" spectroscopy refers to the spectroscopy of a sample that is "in" a given site ("in situ" in Latin), operando spectroscopy denotes spectroscopy of a sample that is "working" ("operando"). 
While in the operando arena UV-vis, Raman and infrared are the most common techniques, others are being increasingly used, such as magnetic resonance spectroscopies [41-43]: EPR spectroscopy can provide important information on paramagnetic species with one or more unpaired electrons and thus characterize the structural and electronic properties of transition metal ions; meanwhile NMR characterizes the chemical environment, structure, and dynamics of molecules. NMR is particularly informative to both catalyst state and reaction progress in time and space. More specialized synchrotron-based spectroscopic and non-spectroscopic techniques are quickly developing to perform operando experiments with high resolution [44], often including complementary vibrational spectrometers as well [45]. Operando methodology has also been developed to operate with techniques such as neutron scattering [46, 47], and the field of techniques adapted to the harsh operando conditions for visualizing atomic-scale properties of active catalysts under actual working conditions is continuously expanding [48, 49]. For instance, major progress with differential pumping has allowed performing TEM and XPS analyses at conditions that increasingly resemble real catalytic operation, under pressures of some mbar. More recently, graphene windows are used as monoatomic frontier to preserve the vacuum needed for TEM or XPS analyses while following reactions in the liquid phase or at atmospheric pressure [49].
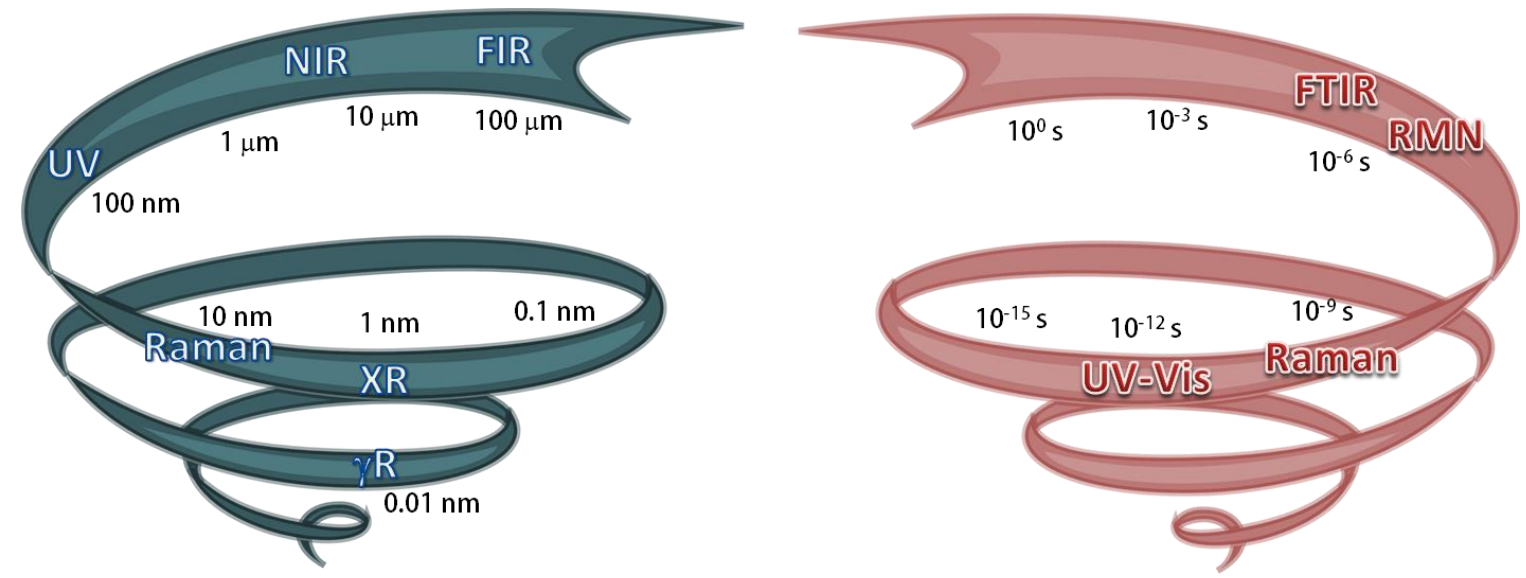

Figure 2. Overview of the spatial (left) and temporal (right) resolution of vibrational microspectroscopic techniques applied in heterogeneous catalysis.

The use of diverse spectroscopies provides not only different sensitivities, but also different time- and space- resolution of measurement, as shown in Figure 2. Space-time constraints are deeply entangled and they are critical for implementing comprehensive operando mapping of catalysts. Higher space-resolution requires better time-resolution to be significant. Moreover, to fully overcome the "dimension gap", besides reducing the size of the area to be probed (from which the information is averaged), probing various areas of the solid is essential, in order to be able to identify non-uniform features and their effect on the catalytic performance. The choice of space resolution defines the perspective of the look: space-resolved operando is nowadays applied at the nano-, micro-, and macro-scale to study intra- and inter-reactor -catalyst and -molecule phenomena and their evolution, often with the help of computational data treatment. The evolution and achievements of imaging in heterogeneous catalysis at different length and time scales has been greatly pushed and reviewed by the group of Weckhuysen [44, 51, 52] and others [29]. The development of microscopic methods and of capillary and fiber optic probes has greatly 
contributed to improve space-resolution [53], but also to perform multi-probe characterization. An interesting example is the operando study of the $\mathrm{CrOx} / \mathrm{Al}_{2} \mathrm{O}_{3}$ extrudates deactivation in a pilot-scale propane dehydrogenation reactor. The experimental set-up combined UV/vis and Raman optical fiber probes to follow the formation of coke deposits on-line [54]. A flow of $\mathrm{N}_{2}$ was used to cool the Raman probe, protecting the optics and Teflon O-rings, and to keep the tip clear of coke deposits. The probe could be moved with respect to the catalyst bed for focusing purposes, and the signal of the Si-O vibrations of the fiber was filtered. The diffuse reflectance $\left(R_{\infty}\right)$ of the catalyst obtained by UV/vis spectroscopy was used to correct the Raman signal for the self-absorption effect due to catalyst darkening. This approach provided a monitoring tool to minimize aging due to thermal stress by decreasing temperature peaks on reoxidation and also to modulate the feed to keep the catalyst working around its optimum performance $[33,54]$.

In summary, the operando spectroscopy methodology has been adopted by catalyst scientists even before, but especially since the term was coined in 2002 [27, 55-57], and the interest and success of this methodology is exponentially increasing due to its ability to establish fundamental molecular structure-activity/selectivity relationships. This is reflected in the literature on catalysis by circa 100 operando publications per year [58], the "In-situ characterization of heterogeneous catalysts" themed issue of Chemical Society Reviews guest-edited by B. Weckhuysen in 2010, and many other recent reviews [17, 53, 58]. Major research effort is currently devoted to investigating all kinds of catalytic systems, and thus new set-ups are being developed to obtain a) more realistic conditions not altered by the probe ( $\mathrm{P}, \mathrm{T}$, environment, mass and heat transfer limitations, see for example, [25, 59] b) better time-resolution,[60] c) better space-resolution in the solid [61] and fluid $[62,63]$ phases, and d) complementarity, coupling several techniques to obtain full characterization data.[64]

\section{Chemical structure and activity in the operando methodology}

Operando methodology stands on two pillars: chemical structure and reactivity. Both must be accurately determined.

\subsection{Catalytically relevant chemical structures}

For all catalyst characterization techniques, a premise remains fundamental: the chemical phase relevant to the catalytic process must be identified. Heterogeneous catalysis is an extremely surface-sensitive phenomenon, the interactions occur at the outermost layer of the catalyst, and the characterization has to be related to the exposed surface (external or internal) of the solid to be representative of active sites. This brings an important conceptual constrain for operando investigations: they must characterize the chemical structure that is relevant to the reaction and measure the activity that is derived from it. However, most spectroscopies are bulk sensitive or near-surface sensitive. Most characterization techniques present signals with contributions from both the bulk and the surface, only very seldom are sensitive only to the outermost layer. The interplay between the bulk and the outermost domains can be critical for heterogeneous catalysis. Two extreme examples would be molecularly dispersed catalysts and bulk catalysts. The former are characterized by having the active phase totally exposed at the surface; the characterization of the dispersed molecules or atoms corresponds to domains exposed to reactants, and therefore possibly relevant. The latter possess an exposed surface and an 
unexposed bulk domain, with a surface to volume ratio that depends on the particle size; as we go "nano", the surface becomes dominant. Large bulk particles will provide an overwhelming signal from the bulk domain that precludes analyzing the evolution of the signal from the outermost active layer during reaction. A more convenient approach is the use of support-stabilized or dispersed nanoscaled particles. The stabilization/immobilization by a support, as in the case of supported mixed molybdates [65] or antimonates [66], minimizes safety issues related to nanoparticles exposure [67]. Moreover, in the absence of a stabilizing support, sintering processes during nanoparticles reaction would preclude the benefit of a higher surface-to-volume signal ratio.

Supported oxides provide a nice example where it is possible to assess the interaction between the reacting molecules and the dispersed phase, free of interference from bulk phase signal from the active component, and ideally also from the support. Figure 3 shows the operando Raman-GC study during propane dehydrogenation on alumina-supported vanadia [68]. $\gamma$-alumina has no Raman bands; the mode at $1012 \mathrm{~cm}^{-1}$ indicates that alumina-supported vanadia remains essentially oxidized and dispersed during oxidative dehydrogenation $(\mathrm{ODH})$. When the reaction switches to non-oxidative dehydrogenation (DH) the hydrocarbon reduces vanadia progressively; once vanadia is reduced, carbon deposits build on the surface. Upon reoxidation, carbon deposits are burnt-away and the oxidized supported vanadium oxide species are restored.
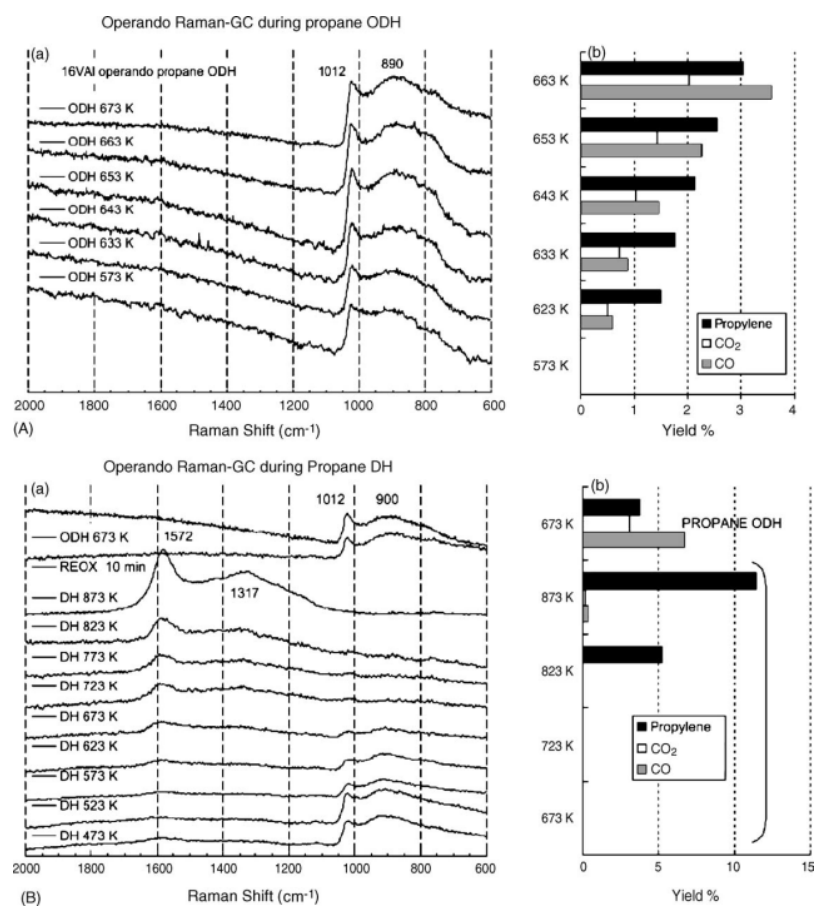

Figure 3. Operando Raman-GC study of a $\mathrm{VOx} / \mathrm{Al}_{2} \mathrm{O}_{3}$ catalyst. Raman spectra at different reaction temperatures (left) and activity data vs. reaction temperature (right) during (TOP) 
propane $\mathrm{ODH}$, and (BOTTOM) propane $\mathrm{DH}$ and in situ reoxidation at $773 \mathrm{~K}$. Catalyst weight: $150 \mathrm{mg}$, total flow: $67 \mathrm{ml} \cdot \mathrm{min}^{-1}$, reaction feed: propane/oxygen $/$ helium=1/6/4 for $\mathrm{ODH}$ and 1/0/6 for $\mathrm{DH}$. Reproduced with permission from [68].

\subsection{Relevant catalytic activity data}

The very concept of operando methodology requires the simultaneous characterization of reactants, products and catalyst during operation. Not only the available characterization techniques have been adapted to the reaction conditions of the operando study, but also the reactors themselves have been modified in the research laboratories to serve as reactors and cells which provide high quality data and allow a variable range of gas hourly space velocities (GHSV) to play with the conversion factor.

\subsubsection{Kinetically relevant reactor cells}

Most operando reactor-cells follow the original idea [55] of obtaining kinetically relevant activity data, so that conversion and selectivity values are similar to those delivered by the original reactor [69]. For example, Figure 4 shows an operando fixed-bed reaction-cell and the ethane conversion Arrhenius plots obtained for a ceria-supported vanadia catalyst during successive runs. The catalyst undergoes significant structural changes in the temperature range. The activity data linear fits of the fresh and the deactivated catalyst indicate that they have the same apparent activation energy (slope), and thus the nature of the active sites would not be modified, although their number (pre-exponential factor) would be reduced upon deactivation. The operando approach allows spotting which functional group must be the active site, namely the $\mathrm{V}-\mathrm{O}-\mathrm{Ce}$ bond.
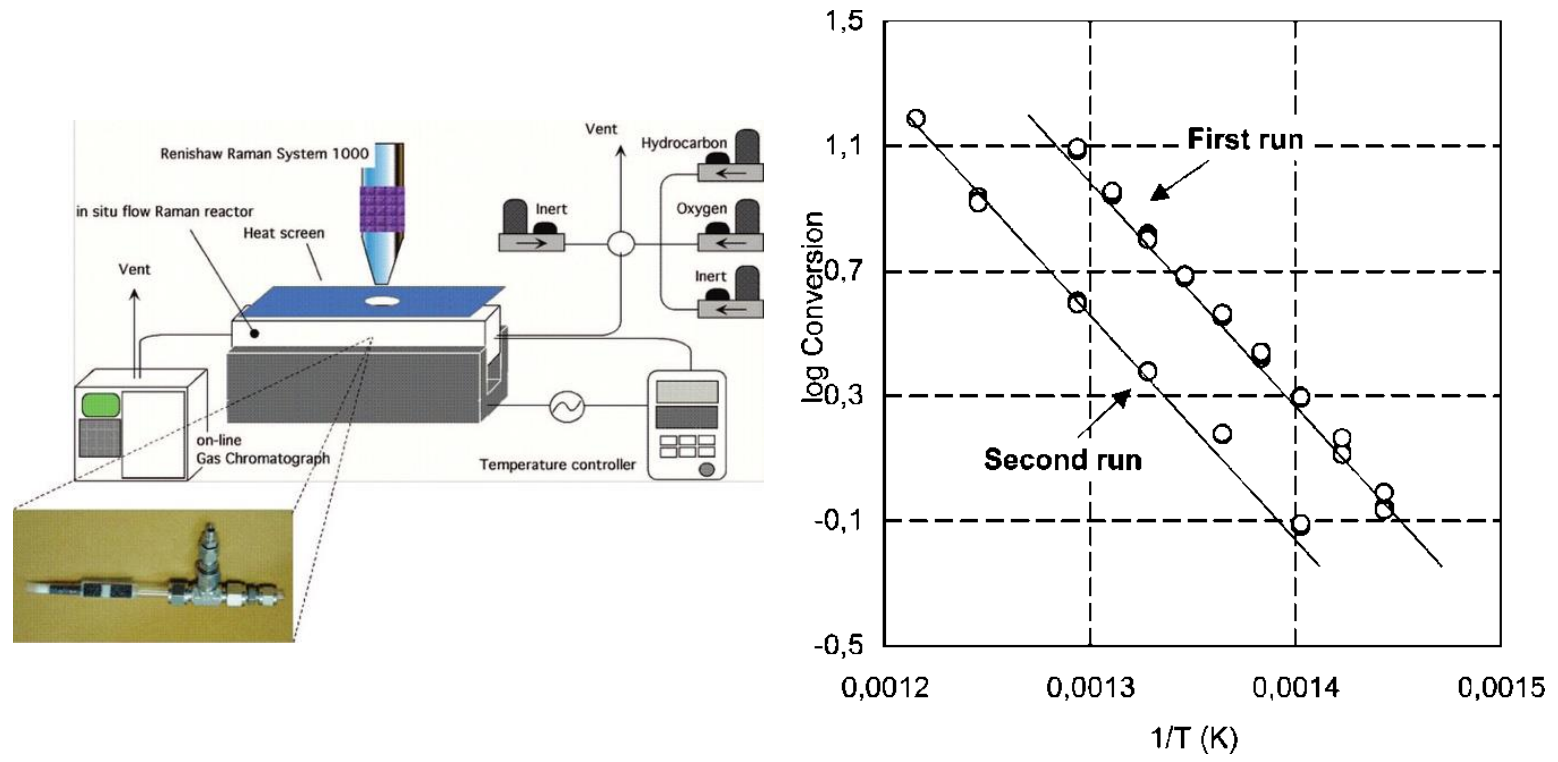

Figure 4. Arrhenius plots obtained in an operando fixed-bed reactor using Raman spectroscopy with gas cromatography during ethane oxidative dehydrogenation on vanadium oxide molecularly dispersed on ceria. Reproduced with permission $[68,70]$.

Operando reactor-cells must comply a minimum of characteristics to obtain kinetically relevant activity data, such as minimizing the dead volume, dissipating any heat generated due to hot spots (to be able to work in isothermal conditions), providing good contact 
between reactants and catalyst, or avoiding reaction intermediates reabsorption phenomena. However, some reaction cells have void volumes or preferential ways that can compromise the validity of the catalytic data. That may be the case of diffuse reflectance spectroscopy cells, which can be used for UV-vis, infrared or X-ray spectroscopy. In 1994-1996, Wolf and Fehlner already reported the use of a modified Harrick diffuse-reflectance infrared Fourier-transform spectroscopy (DRIFTS) reaction cell: "The DRIFT cell was designed so that the gases will flow into it from beneath the sample and through it, ensuring an appropriate contact between the gases and the catalysts." The DRIFT cell was optimized for quantitative catalytic measurements by providing spacing below (upstream) the fixed bed to distribute gas flow [71-73]. A very detailed analysis and fluid dynamics optimization in DRIFT cells was reported recently by Meunier [25].

Microstructured reactors typically satisfy the above requirements, since they allow good control of heat and mass transfer and hydrodynamics and permit facile access to spectroscopic catalyst characterisation. In addition, they have shown to inhibit lightoff and hysteresis effects as compared to bigger reactors. Furthermore, microstructured channel reactors allow easy and modular integration of other functional units (e.g. mixing, separation) and also the incorporation of planar waveguides and optical fibres [7].

In the case of macroreactors care must be paid, especially if we want to mimic industrial requirements (temperature and pressure, GHSV, type of bed, geometry of the catalyst, etc.), which are essential in the final efficiency of the catalyst and may mark and define the conditions that must be adopted for operando methodology. Plug-flow catalytic reactors operating at low conversion values will have rather limited changes in the flow composition and thus the environment can be assumed to be homogeneous in the whole catalyst bed. In these differential reactors, activity data obtained in the reactor outlet can be used to calculate reaction rates and correlated with structural characterization obtained at any position of the catalytic bed. However, low conversion is usually not representative of true industrial conditions, and relevant effects may go unnoticed or be misunderstood under this controlled operation. In integral-type reactors, operation may be in stationary state in time, but there may be significant gradients in space. Thus, resolution along the axial and often radial directions is crucial for gaining insight into the mechanisms and optimizing the performance, because prominent concentration and temperature gradients, and thus structural changes, exist [74].

\subsubsection{Transient conditions}

Steady-state reaction conditions are typically used in kinetic investigations; however, further insight can be obtained under transient conditions. Step/pulse-response experiments where one variable is modified (concentration, temperature, irradiance, etc.) help to increase the detection sensitivity and selectivity. Especially useful for operando studies is the steady-state isotopic transient kinetic analysis (SSITKA), because microstructure and reactivity are not altered by the isotopic exchange. SSITKA studies have allowed demonstrating mechanistic aspects, such as the role of species as real reaction intermediates or simple spectators. For instance, Meunier et al. [75] demonstrated that surface formate and carbonate species were not reactive intermediates during watergas shift (WGS) reaction on $\mathrm{Au} / \mathrm{Ce}(\mathrm{La}) \mathrm{O}_{2}$ catalyst at $155^{\circ} \mathrm{C}$ (Figure 5 ). 

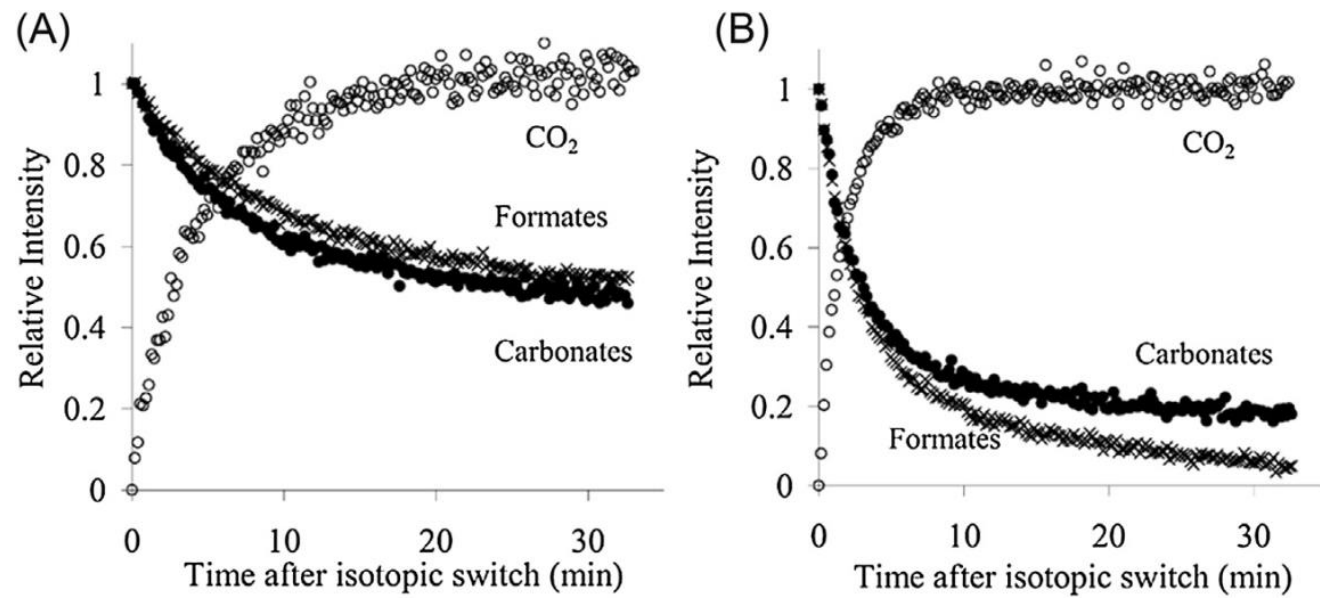

Figure 5. Relative evolution of the FTIR bands of ${ }^{12} \mathrm{C}$-containing carbonate, ${ }^{12} \mathrm{C}$-containing formate and ${ }^{13} \mathrm{CO}_{2}$ at $155{ }^{\circ} \mathrm{C}(\mathrm{A})$ and $220{ }^{\circ} \mathrm{C}$ (B) under $2 \%{ }^{13} \mathrm{CO}+7 \% \mathrm{H}_{2} \mathrm{O}$, following steadystate under $2 \%{ }^{12} \mathrm{CO}+7 \% \mathrm{H}_{2} \mathrm{O}$. Reprinted with permission from [75].

Another approach reported by Thibault et al. used fast pressure jumps and microsecond infrared spectroscopy to monitor adsorbed $\mathrm{CO}$ as a probe molecule [76]. This was used to investigate the accessibility and evolution of platinum nanoparticles supported on ZSM-5; as Figure 6 reflects, they identified different sintering mechanisms in the mesopores and the zeolite external surface.

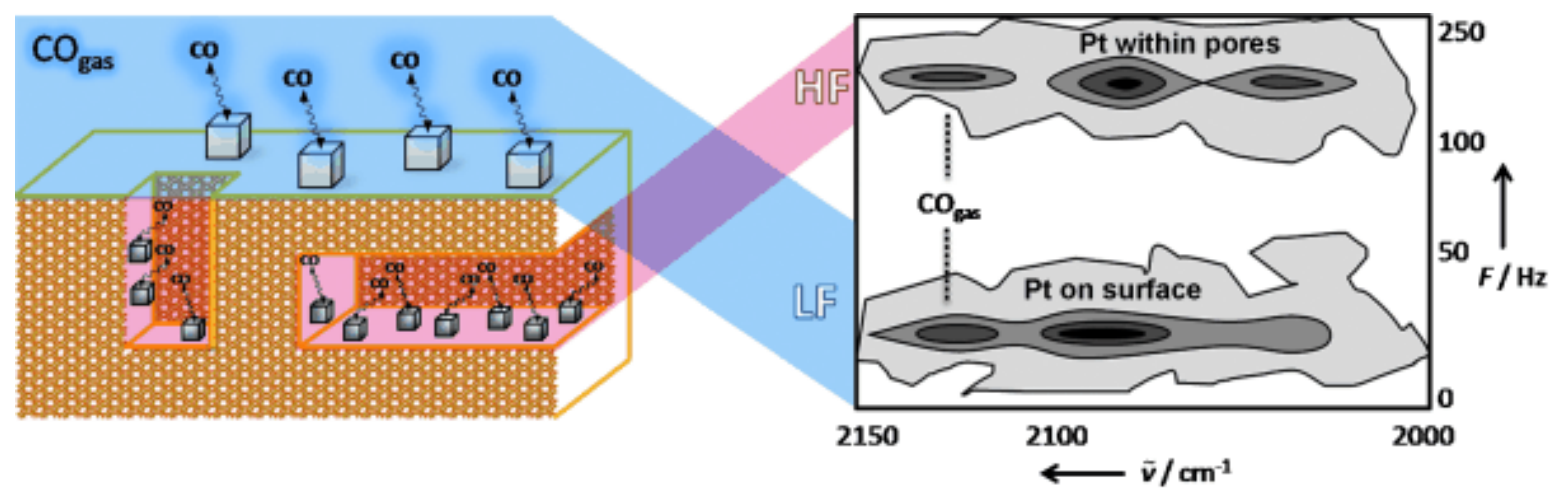

Figure 6. Sintering mechanism on Pt/ZSM-5 catalysts on the external surface and in the mesopores according to 2D IR pressure-jump spectroscopy of adsorbed CO. Reproduced with permission from [76].

\subsubsection{Wafer catalyst}

Geometries other than conventional fixed-bed reactors may be required in some operando set-ups even though they may alter the reactor fluid dynamics, which is critical for kinetically relevant activity measurements, and/or their preparation may alter the textural properties of the catalyst. This is the case of thin wafers, which may be useful to represent a honeycomb wall or to allow spectroscopic techniques operation in transmission mode, as in the case of X-ray absorption (XAS) [77, 78] or infrared absorption spectroscopy. 
The use of a wafer-shaped catalyst is particularly demanding from the operando perspective because the performance must be free of diffusional limitations. There is thus a dual requirement: (i) the need for efficient gas flow distribution, and, (ii) efficient access of reactant to the active sites.
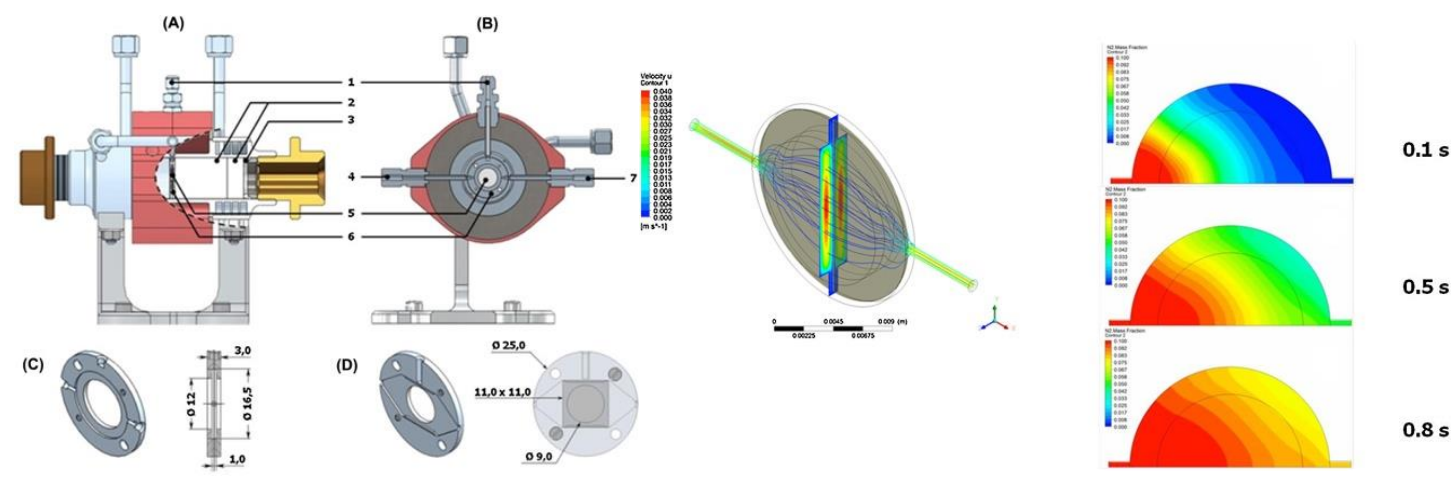

Figure 7. Left: 'Sandwich' IR reactor cell $(\mathrm{A})$ longitudinal, and $(\mathrm{B})$ radial views. 1 Thermocouple location, 2 - KBr windows, 3 - O-ring, 4 - Gas inlet, 5 - Sample, 6 Sample holder, 7 - Gas outlet. (C) Circular, and (D) square sample holders. Right: Flow model in the round sample holder (left panel) and evolution of the concentration with time (right panel). Reprinted with permission [59]

(i) On the need of efficient gas flow distribution: the design of kinetically relevant transmission infrared catalytic cells has been a constant challenge in the past years [59, 79 , 80]. Daturi's group has reported an accurate design with gas flow optimization for wafer catalysts, the so-called "sandwich" cell (Figure 7). Flow simulations concluded that the flow regime in the 'sandwich' IR reactor cell is a combination of ideal plug-flow reactors both in series and parallel. An operando SSITKA study of methanol adsorption onto ceria as model probe reaction pointed out the relevance of the sample holder design and the geometry of the catalyst to obtain reliable kinetic results [59]. A square pellet in a squareshaped sample holder led to a simpler flow distribution and a more accurate kinetic model than a round wafer.

(ii) On the efficient access of reactant to the active sites: in general it should be kept in mind that using operando cells will always be a compromise between optimal spectral quality and reliable activity data [77]. In this sense we must also highlight the effect that pelletization may cause in diffusional phenomena. The pelletizing process inevitably modifies the porosity of the sample (see Figure 8). An excessive pressure in the pelletizing process to obtain a thin wafer from powders will modify the porous structure of the solid and it is well-known that the pore size distribution affects the catalyst efficiency. Macropores will disappear in exchange for a greater contribution of meso- and micro-pores in the wafer. This shift towards smaller pore sizes may hamper the catalytic efficiency. This phenomenon can also apply to conventional activity measurements when samples are too fine and need to be pelletized and crushed to obtain adequate particle size fractions for the catalytic bed. 


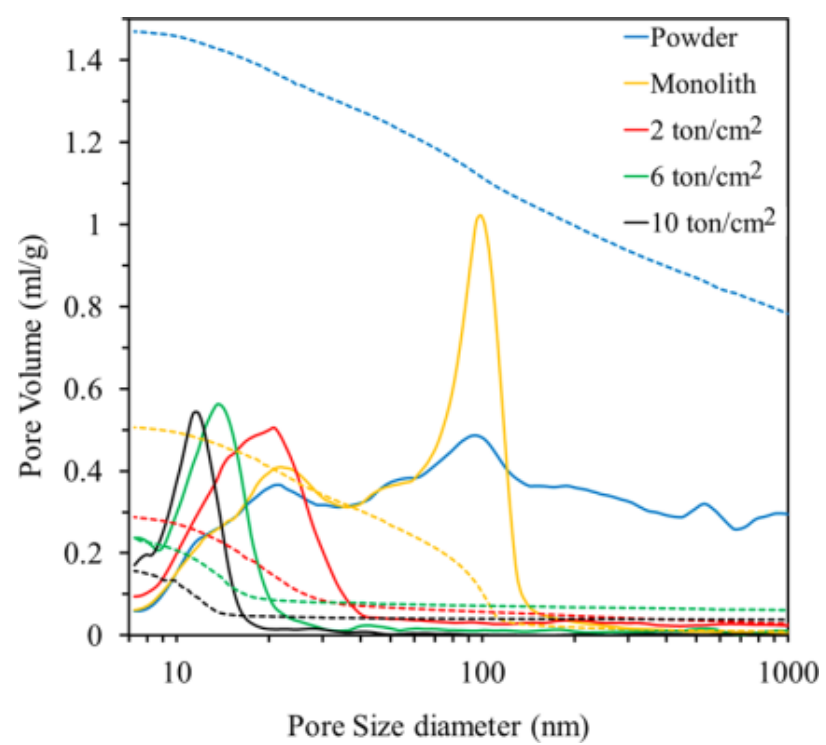

Figure 8. Pore size distribution (line) and cumulative volume (dotted line) for uncompacted and compacted titania-supported vanadia-tungsta catalyst at different pressures [81].

Gas molecules will enter in the small pores, hit and bounce on the walls and consequently, they will diffuse more slowly. In case of sufficiently small catalyst particles, the intraporous diffusion path is shorter and may be enough to allow uniform access of the reactants to all the active sites. However, in pellets, the intrinsic reaction rate can be faster than the diffusion rate inside the catalyst wall. The reactants are then used up within a thin superficial layer of catalyst, while most of the inner catalyst wall remains inactive. The directly proportional relationship between the Thiele modulus and the thickness of the pellet can be used as a simple procedure for evaluating efficiencies on the basis of pellet dimensions and solid phase characteristics [81]. Figure 9 shows the efficiency of a titaniasupported vanadia-tungsta catalyst pressed into a self-supported wafer for NOx selective catalytic reduction in an operando infrared transmission reactor. The wafer thickness and the pressure applied to make it have a dramatic effect on the efficiency number. Accurate operando studies require careful control of the effect of pelletizing for a correct calibration of the operando reactor.

Summarizing, the pelletizing procedure may have a relevant effect on the activity, not only for transmission FTIR operando set ups, but also for general catalytic measurements, operando studies should be well aware of the dramatic role of porosity on the reliability of activity data. Spectroscopy, fundamental catalysis and chemical engineering meet at the operando approach to understand catalysis. 


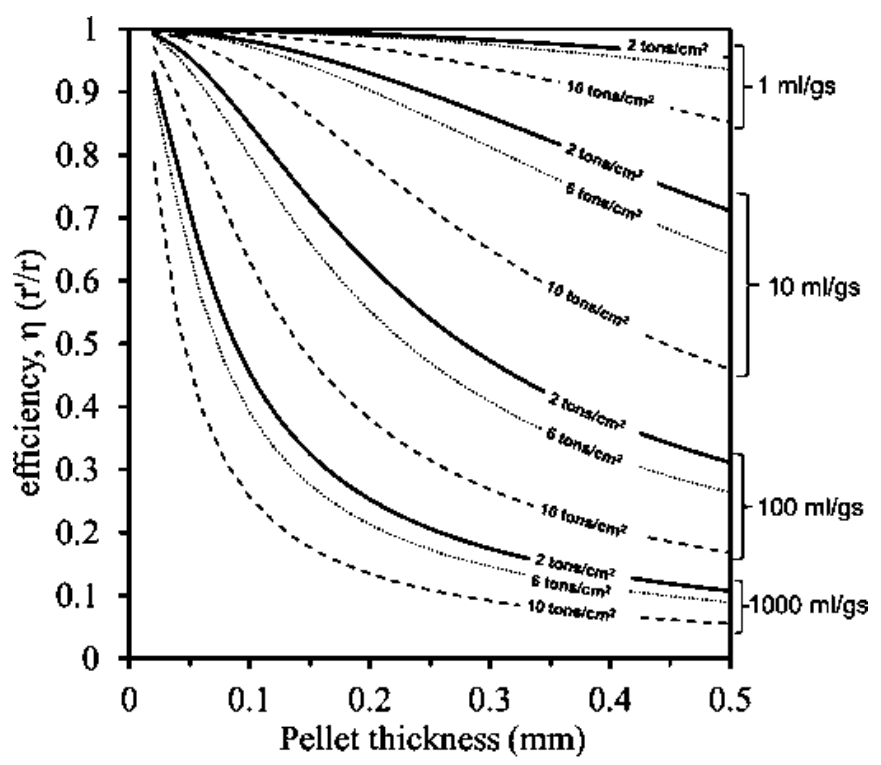

Figure 9. Effect of pelletizing pressure and wafer thickness on catalyst efficiency and intrinsic activity during NOx selective catalytic reduction on titania-supported vanadiatungsta wafers in an operando transmission IR reactor. Reproduced with permission from [81].

\section{Engineering operando methodology}

The progress in the reduction of the technical limitations of operando studies has made it possible to go a step forward and tackle the characterization of (micro)structured reactors and solids in their final form, reproducing realistic industrial conditions and helping to bridge the gap between materials science and chemical engineering in heterogeneous catalysis. While fundamental research is mostly carried out with powdered catalysts, the industrial ones are typically shaped. Mass transfer limitations and pressure drop are important factors to be controlled; in addition, all sort of gradients appear: temperature, composition, chemical structure and reactivity. The development of new applications which performance is enhanced by shaped materials is delivering a wealth of benefits whose optimization requires an accurate mapping of those gradients. The next sections will provide an overview on the most recent and/or relevant approaches to bring fundamental science to structured reactors by engineering operando methodology.

\subsection{From powders towards shaped bodies}

The final composition (combination of active phase, support, binder, and additives) and manufacture variables (e. g. extrusion pressure or calcination temperature); and also the thermal, mechanical and fluid-dynamic constraints during operation may significantly alter the properties of the catalyst when it is not a powder, but part of an industrial solid (with beneficial and/or detrimental effects [82]). This greatly justifies an effort for the characterization of unmodified real catalytic bodies during synthesis, activation and reaction, which may provide key information for an increasing number of catalytic systems and reactions of interest. For instance, it may help controlling the selectivity in partial oxidation processes, greatly influenced by contact time and fluid dynamics, or determine the best phase distribution in a monolith (uniform distribution, egg shell, egg white, egg yolk...) [83]. Characterization, however, is usually performed with the powdered form or 
with previously crushed materials [84] or upon peeling off the wash-coated device [85, 86]. The results thus obtained are informative, but incomplete, due to the lack of a relevant dynamic regime and missing information about gradients in space during catalyst operation. Computational modeling and simulation methods have been successfully developed to describe the operation of shaped catalysts (e.g., monolithic structures) in terms of hydrodynamics and mass and heat transfer [87]. However, due to the complexity of combining the requirements of space- and time-resolved solid- and gas-phase characterization with real industrial conditions in reaction cells adapted to the geometry of the catalyst, the application of the operando methodology to functionalized bodies in the final industrial form has been only scarcely attempted. Over the past two decades, a large research effort has been devoted to the development and optimization of analytical tools. This has mainly used vibrational and electronic spectroscopies, magnetic resonance, and synchrotron X-ray; these are aimed at resolving the evolution of the chemistry at the macro-, meso- and nano-scale [88] within extrudate pellets, honeycomb monoliths, or foams in operation, but also within powdered packed-bed reactors [89] and microreactors. However, most studies are far from reproducing industrial operation and mostly characterize small extrudates during synthesis stages or in designed reaction experiments, or do not include activity data.

\subsection{Local operando studies in integral reactors}

In integral reactors, the spot of measurement is of paramount importance, but obtaining spatial resolution requires technical developments difficult to implement for some analytical tools and/or geometries. In these cases, single-point operando characterization of integral catalytic reactors may provide useful information overlooked with the "black box" approach or during operando studies with non-representative configurations (for instance with altered fluid dynamics). In this line, Daturi's and Bañares' groups have recently reported the first attempts which deal with vibrational spectroscopy operando studies of honeycomb monoliths, demonstrating the reliability of the devices shown in Figure 10.

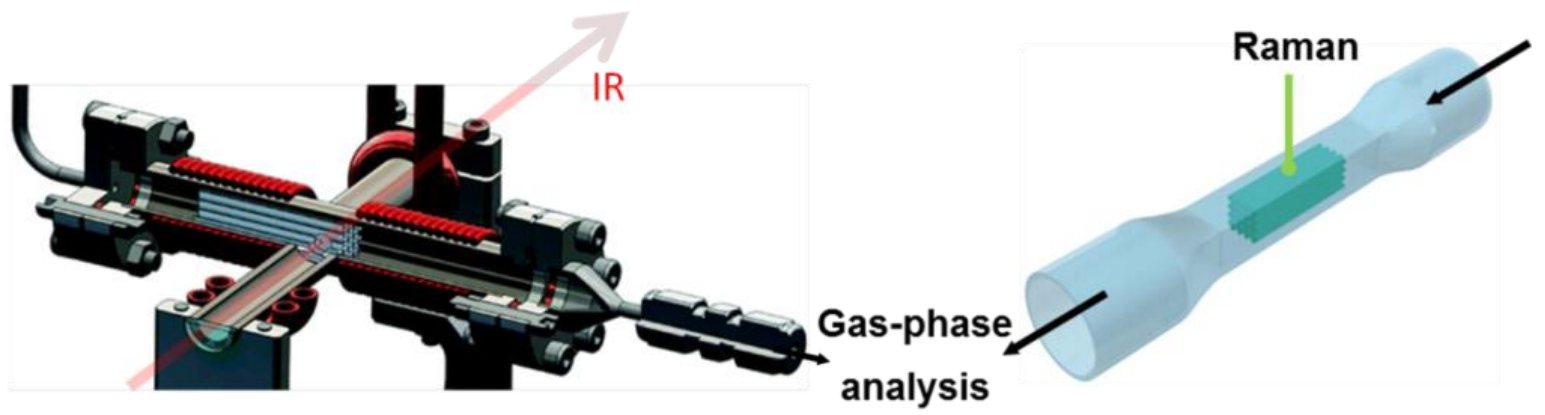

Figure 10. Vibrational spectroscopy operando reaction cells for monoliths. Left: transmission FT-IR, reproduced with permission from [39]. Right: Raman, reproduced with permission from [15].

$\mathrm{A} \mathrm{V}_{2} \mathrm{O}_{5}-\mathrm{WO}_{3} / \mathrm{TiO}_{2}$-sepiolite monolithic catalyst was monitored by FTIR-MS for $\mathrm{NH}_{3}-\mathrm{SCR}$ as case study to validate the transmission FT-IR set-up for honeycombs [39]. A hole was drilled through all the monolith walls except for one, which was polished to allow IR beam transmission. A number of characteristic bands were detected in the MIR region. The higher sample thickness of the integral reactor, compared to a typical wafer, and the presence of the sepiolite clay lead to saturation in several spectral ranges (3000 to 3500 
$\mathrm{cm}^{-1}$ and below $1250 \mathrm{~cm}^{-1}$ ), hampering accurate analysis of adsorbed ammonia bands. The assignment of the band at $1600-1650 \mathrm{~cm}^{-1}$ is not straightforward because of the overlapping contributions of $\delta \mathrm{OH}$ and $\delta_{\text {as }} \mathrm{NH}_{3}$; this difficulty was overcome by Rasmussen et al. performing a chemometric analysis [90]. They showed that on a $\mathrm{V}_{2} \mathrm{O}_{5}-\mathrm{WO}_{3} / \mathrm{TiO}_{2}-$ sepiolite honeycomb with pre-adsorbed ammonia, it is possible to study the evolution of ammonia and ammonium surface species (infrared) with respect to NO selective catalytic reduction reaction (online mass spectrometry). Both ammonia-related species, ammonia on Lewis acid sites and ammonium on Bronsted acid sites, appear involved in the reaction. The interplay was further analyzed in a high time-resolution operando transmission infrared cell on a wafer catalyst, showing that SCR activity is much higher when adsorbed ammonia reacts with water formed in reaction to create adsorbed ammonium species (Figure 11).

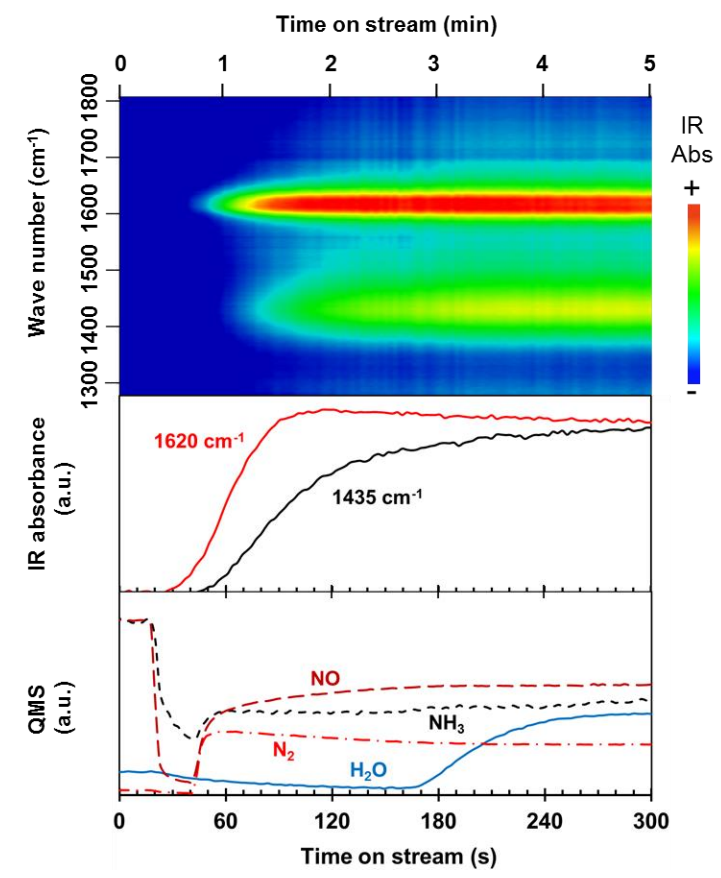

Figure 11. Transient state operando study on a $\mathrm{V}_{2} \mathrm{O}_{5}-\mathrm{WO}_{3} / \mathrm{TiO}_{2}$-sepiolite wafer catalyst. TOP: FTIR spectra contour plot showing the growth of ammonia-derived species on the surface after changing from $20 \% \mathrm{O}_{2} / \mathrm{Ar}$ to SCR conditions. MIDDLE: FTIR area profiles of $\mathrm{NH}_{3, \text { ads }}$ and $\mathrm{NH}_{4}{ }^{+}$. BOTTOM: evolution of gas phase species concentration measured by MS. Reproduced with permission [90].

Propane ammoxidation was the probe reaction used to assess the role of catalyst shape with the Raman system for monolithic reactors [15]; the behavior of an alumina-supported vanadium phosphorous oxide (VPO) catalyst shaped as a monolith was compared to that of the corresponding powdered fixed bed. The lower effective thermal conductivity and pressure drop of the honeycomb structure together with the different textural properties result in different performance. The conversion of oxygen compared at any given conversion of propane is lower for the packed-bed reactor configuration; thus, the operando Raman spectra show the formation of VPO phases there, while dispersed vanadia is apparent on the honeycomb configuration. The value of this approach is to reach a relationship between the chemical structure and performance of a given catalyst formulation depending on its shape (powder vs. honeycomb). 


\subsection{Space-resolved operando studies}

The characterization of several areas of a working solid to obtain a time-resolved profile can be approached by sequential single-point characterization (with moving catalysts/reactors or probes), by simultaneous multi-point characterization to reduce the "time gap", or, the best space-resolved option, by a technique+detector combination capable of probing a surface (2D imaging). Then a 3D picture (tomography) can be obtained by displacement of the sample. The whole characterization can be performed in just one experiment if the process under study is in steady state or slow enough, or if the measurement+displacement is fast enough. Otherwise, for easily reproducible fast processes the whole profile/picture/tomography can be reconstructed from several experiments measuring each time at a different location; spectroscopic methods with adequate temporal resolution are required to follow with space resolution the dynamics of ignition or extinction of reactions; catalyst formation or activation; or transient or cyclic phenomena. Grunwaldt et al. [91] presented an overview demonstrating that time-resolved and spatially resolved spectroscopic studies are important for understanding the preparation of catalysts and the structural changes under dynamic conditions (e.g. activation and start-up) and reaction conditions.

In integral reactors, not only the catalyst microstructure should be characterized with spatial and temporal resolution, but also the fluid phase composition and the temperature in the solid and fluid phases, because they may provide useful information. The ability to simultaneously probe both the gas and solid phases of the heterogeneous catalytic system with several space-resolved techniques will draw a highly defined picture of the processes taking place. However, the technical difficulty is high and the number of probes, the spaceand time-resolution, and/or the reproducibility of true industrial conditions is usually limited. A recent and complete review covers the spatial-resolution operando methodologies applied to catalysts and catalytic reactors [53], with careful emphasis on assessing the invasive nature of physical sampling probes and the enabling capabilities of spaceresolved operando to improve kinetic modeling. The following sections are an overview of the advances towards operando studies applied to structured reactors with space-resolved characterization of the solid microstructure, the fluid composition and the temperature.

\subsubsection{Mapping solids during operando studies}

Movable/multiple probes connected by optical fibre to the spectrometer can be used with DRIFTS [92], UV-vis-DRS [93] and Raman [94] to characterize with space resolution (within a limited spectral and thermal range). In the literature on operando vibrational spectroscopy studies only a couple of examples get spatial resolution by displacing the sample/reactor using a motorized support. Figure 12 shows how the combination of both surface-sensitive (DRIFTS) and bulk-sensitive (Raman) time-resolved detection at three different catalyst-bed positions $\left(2.5 \mathrm{~mm}\right.$ distance) during $\mathrm{NO}_{x}$ storage and reduction on Pt$\mathrm{Ba} / \mathrm{CeO}_{2}$ was useful in establishing a delay in the formation of nitrite and nitrate surface species, and also a decrease in their surface coverage downstream [18]. 

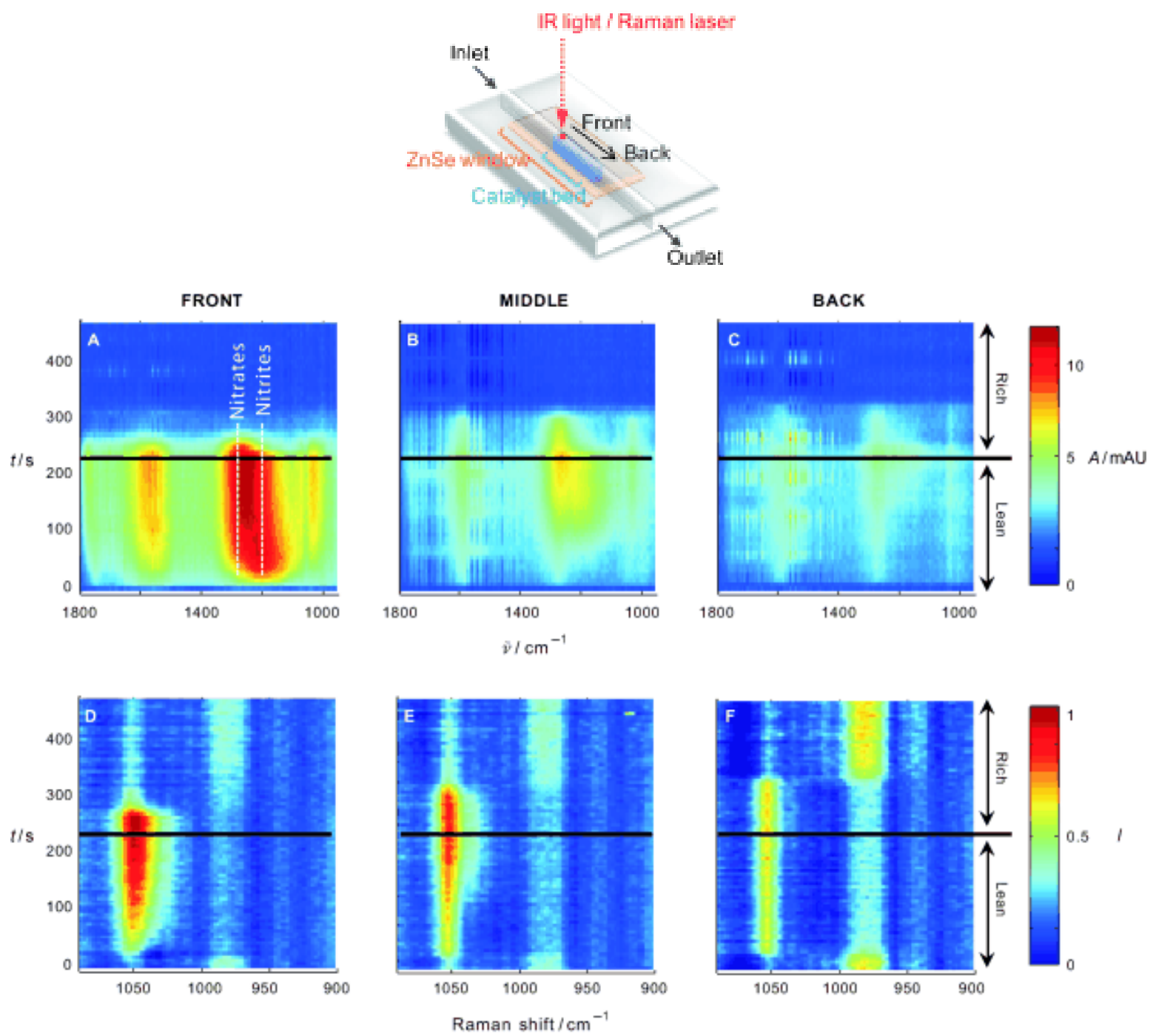

Figure 12. (TOP), schematic illustration of a cell for space- and time-resolved DRIFTSRaman experiments. (BOTTOM), DRIFT (A-C) and Raman (D-F) spectra during NOx storage reduction (NSR) at the front, middle, and back positions of a Pt$\mathrm{Ba} / \mathrm{CeO}_{2}$ catalyst bed [18].

Weckhuysen's group reported the use of Diagonal Offset Raman Spectroscopy (DORS), in which the laser and collection optics are fixed at a $90^{\circ}$ angle one to another, so that the spectral contributions of surface and subsurface layers can be separated using multivariate analysis. The sample is moved diagonally and can be translated and rotated. It has allowed for spatiotemporal in situ studies of impregnation, drying, and calcination steps in the preparation of catalytic bodies $[83,95]$ and could be used to monitor them at work. This recently developed technique is an alternative to invasive sample bisection for intracatalyst characterization [92]. In another interesting development, tip-enhanced Raman spectroscopy (TERS) can monitor photocatalytic reactions at the nanoscale [96].

The conventional Raman spectrometer limits the application of this spectroscopic technique in parallel and only sequential detection is possible. However, in the case of FTIR spectroscopy an alternative to common detectors is the focal plane array (FPA) 
detector, which can be used for FT-IR imaging. The advantage is that tens of thousands of spectra can be collected simultaneously with spatial resolution for the measurement of different sections of the monolith or for high-throughput screening of catalysts with parallel operando cells [97].

The use of X-ray microscopy and tomography in catalysis has been thoroughly reviewed by several authors [44, 98]. The combination of micrometrer-length-scale full-field X-ray microscopy, $10 \mathrm{~nm}$-to-100 nm- length-scale scanning X-ray microscopy and atomic-scale electron microscopy covers the whole range of catalyst characterization. For macroscopic operando studies on millimeter-sized bodies hard X-ray with spectroscopic or scattering contrast can provide order information at local (X-ray Absorption Spectroscopy, XAS), short (Small Angle X-ray Scattering, SAXS) and long (Wide Angle X-ray Scattering, WAXS) range, crystalline structure information (X-ray diffraction, XRD), or elemental and density analysis (Compton and fluorescence effects). The technical progress in the field is constant, aided by the development of complex algorithms for data collection and analysis. For instance, the combination of X-ray pair distribution function (PDF) method with computed tomography can overcome the limitation of diffraction techniques to obtain reliable, quantitative, 3D information on the nanostructure of phases without long range order [99]. In order to reduce the space resolution lower energy radiation must be used, and therefore the penetration depth is reduced to dozens of microns (soft X-ray microscopy) or just a few microns (electron microscopy) and the sophistication of reactorcells for operando studies increases. However, these operando set ups are developing fast [48], as well as high-brilliance synchrotron sources and high-quality fast detectors with space and spectral resolution [100], which has led to the construction of hard X-ray microscopes with resolution on the scale from 10 to $100 \mathrm{~nm}$ and potentially below.

Many studies have applied the different X-ray techniques to characterize the active phase distribution in catalytic bodies during the synthesis steps, but less frequently, and only in this decade, to reveal the chemical structure-reactivity relationships during real operation $[77,101]$. A nice example is the dynamic study of $\mathrm{Ni} / \mathrm{y}-\mathrm{Al}_{2} \mathrm{O}_{3}$ extrudates of Figure 13 [102]. First, the 3D profiles constructed from $\mu$-XRD-CT (computed tomography) combined with $\mu$-absorption-CT during the impregnation, calcination in $\mathrm{N}_{2}$ and air, and $\mathrm{H}_{2}$ reduction steps revealed the influence of the complexing agents on the structural evolution and dispersion of nickel during the catalyst preparation and activation. Then, during methanation reaction, they demonstrated the stability of the crystalline metallic Ni fcc component from 200 to 450 ${ }^{\circ} \mathrm{C}$ in terms of phase constitution, spatial distribution and average particle size, and thus concluding that it was the active component of the catalyst. 


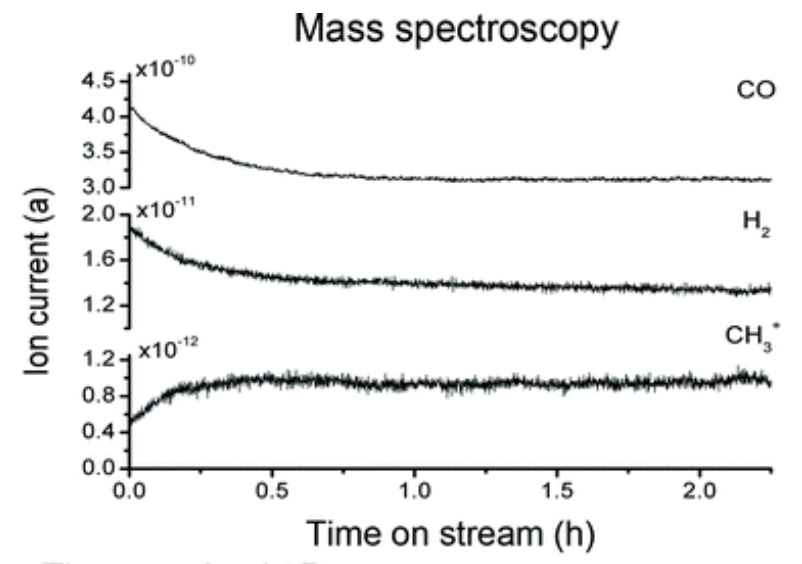

Time-resolved 2D

XRD-CT phase map Summed 1D XRD
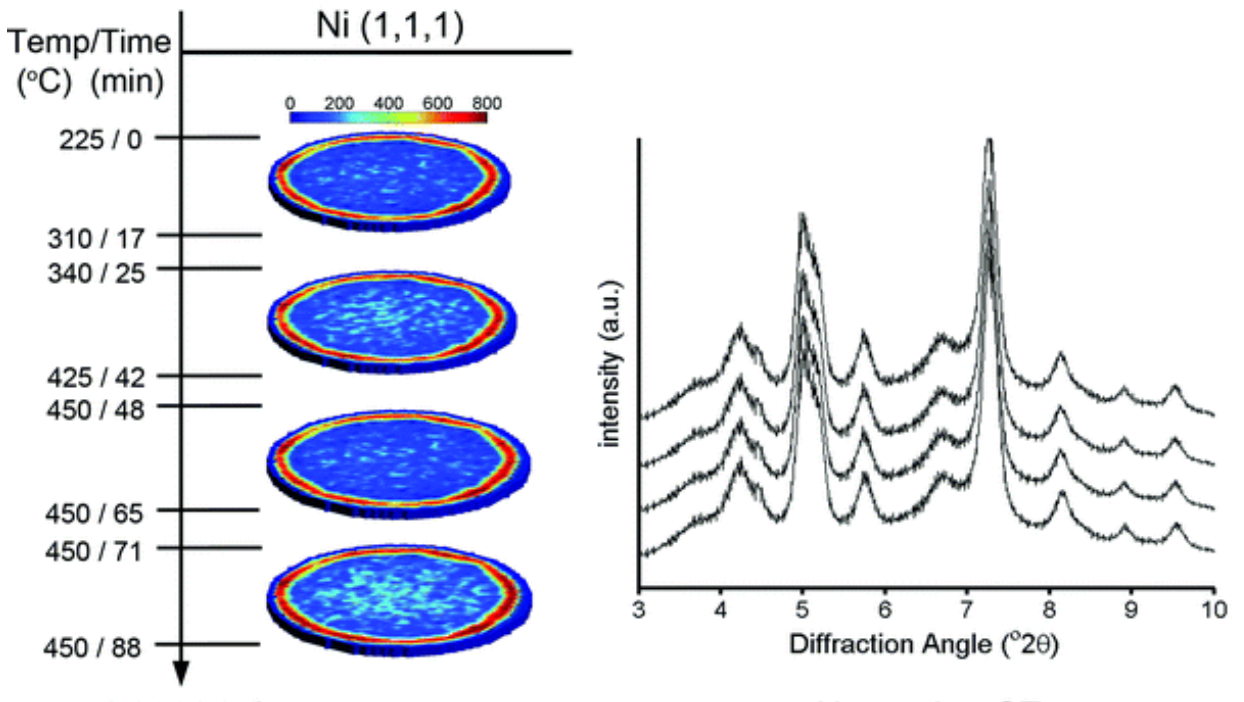

3D XRD-CT Ni phase map

Absorption-CT
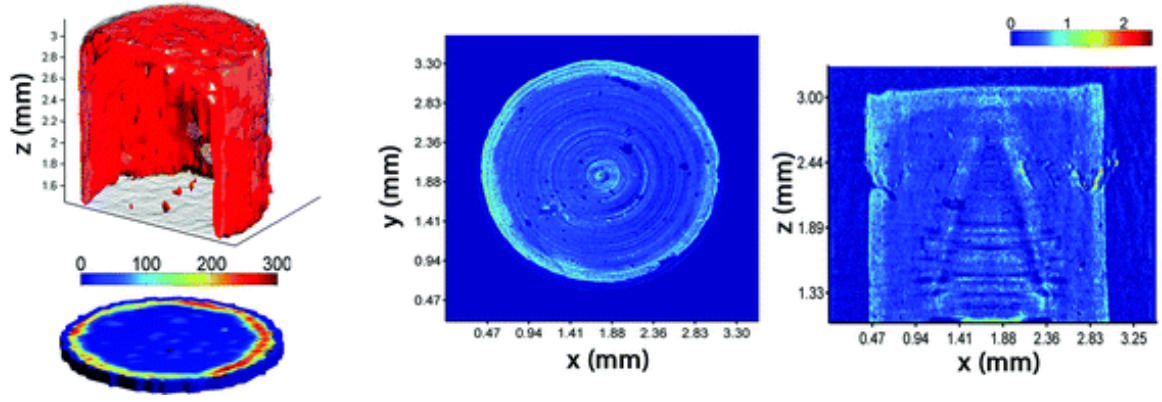

Figure 13. Methanation over $\mathrm{Ni} / \mathrm{Y}-\mathrm{Al}_{2} \mathrm{O}_{3}$ monolithic catalyst. The activity was followed by MS. The stability of crystalline and non-crystalline distributions was proven by $\mu$-XRD-CT (2D during heat ramping, then $3 \mathrm{D}$ ) and $\mu$-absorption-CT. The high quality summed $1 \mathrm{D}$ XRD patterns confirmed that no reaction intermediates were formed. Figures reproduced with permission from [102].

In a very recent work, shown in Figure 14, a $3 \mathrm{~mm} \mathrm{Co} / \mathrm{\gamma}-\mathrm{Al}_{2} \mathrm{O}_{3}$ catalyst was studied by $\mu$ $\mathrm{XRD}-\mathrm{CT} / \mu-\mathrm{PDF}-\mathrm{CT}$ to elucidate the evolution of the Co phases, first during activation (reduction in $\mathrm{H}_{2}$ ) and then under Fischer-Tropsch synthesis conditions. Activity 
measurements were performed using the same reaction conditions as used for the in situ $\mu$-XRD-CT/ $\mu$-PDF-CT study, but not simultaneously [103]. The high volume of data was processed with high-throughput software; their quality allowed following the spatiotemporal dependency of $\mathrm{Co}$ speciation from $\mathrm{Co}_{3} \mathrm{O}_{4}$ to $\mathrm{CoO}$ to face-centered cubic (fcc) Co metal nanoparticles. The complementary data revealed the presence of small nanoparticles difficult to reduce due to high interaction with the support, and agglomerates with weak interaction that are easily reduced. At the sample periphery a significant amount of agglomerated small fcc Co metal nanoparticles oxidize to $\mathrm{CoO} / \mathrm{Co}_{3} \mathrm{O}_{4}$ during reaction coinciding with a decrease in $\mathrm{CH}_{4}$ selectivity and increased water-gas shift activity, which explains the sintering previously observed for such catalysts.

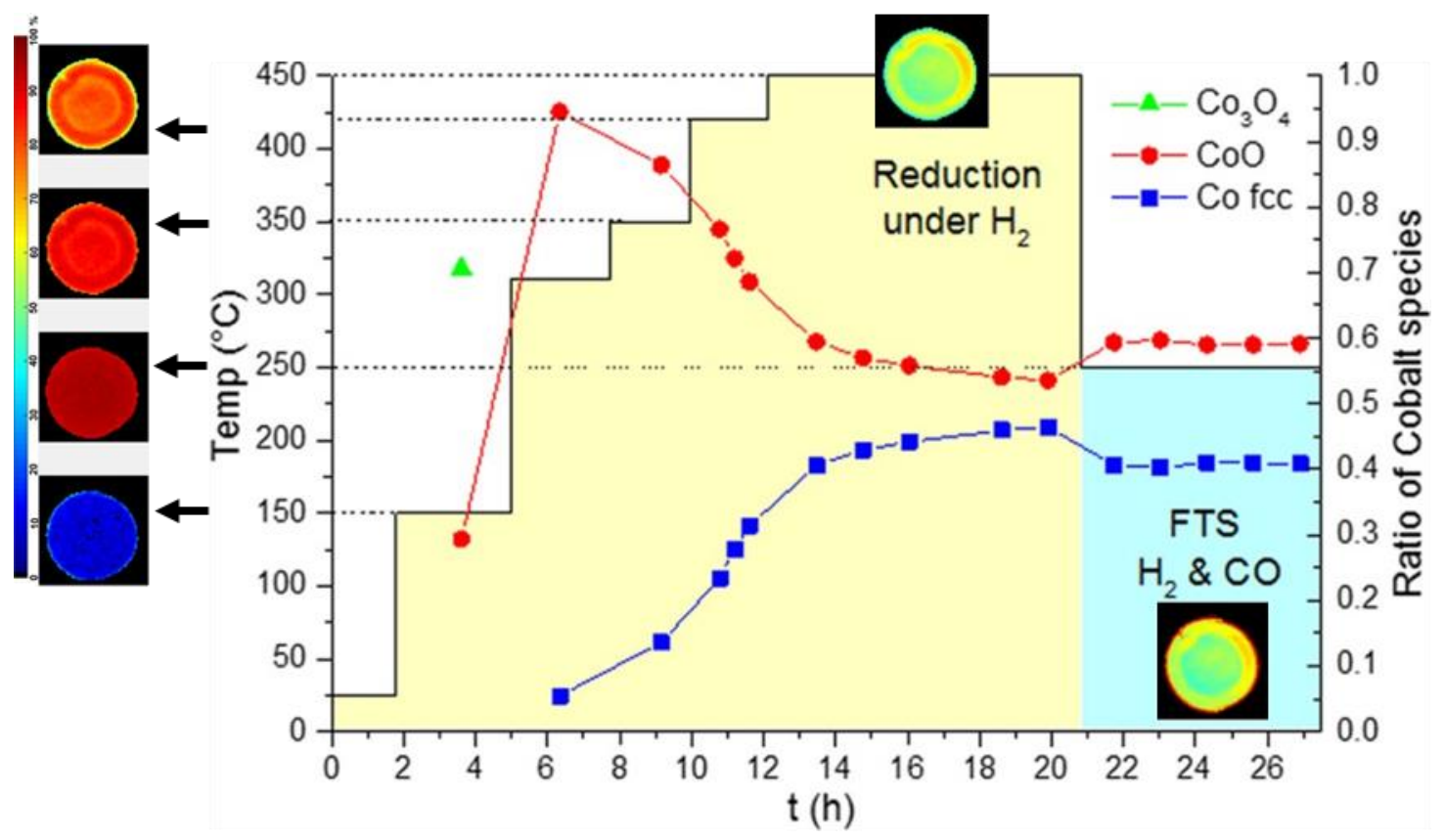

Figure 14. Reconstructed 2D weight percent composition maps of $\mathrm{Co} / \mathrm{Y}-\mathrm{Al}_{2} \mathrm{O}_{3}$ during $\mathrm{H}_{2}$ reduction and Fischer-Tropsch synthesis and composition profiles compiled from the integrated and scaled reflection intensities for the various cobalt-containing phases from the summed 2D diffraction data. Adapted from [103]. Figure cited with permission of ACS as source. https://pubs.acs.org/doi/full/10.1021/acscatal.6b03145. Further permissions related to the material excerpted should be directed to the ACS

The space-resolved operando approach has also been increasingly applied to electrochemical processes. However, in the dynamic imaging studies on fuel cells [104] usually little information is reported concerning the performance of the cell. As a recent example, the distribution and oxidation state of a Pt cathode catalyst in a membrane electrode assembly were followed under a polymer electrolyte fuel cell operating conditions. Computed-tomography imaging with X-ray absorption near edge structure spectroscopy (XANES) combined with X-ray absorption fine structure spectroscopy (EXAFS) revealed the heterogeneous migration and degradation of $\mathrm{Pt}$ during an accelerated degradation test [105]. In another fuel cell, especially designed for the study, phase contrast X-ray imaging has been employed to obtain a 3D quantitative distribution of the very low water content within the $\mathrm{nm}$-sized pores of carbon-based microporous layers 
[106]. Also, a simplified binder-free model high-performance Li/S cell was designed to monitor at work a $\mathrm{N}$-doped reduced graphene oxide/carbon monolith used as cathode [107]. The operando experiment combined electrochemical impedance spectroscopy (EIS) and X-ray radiography characterization during galvanostatical charge/discharge cycles. Several macroscopic effects were observed: the formation and disappearance of centimeter-long sulfur dendrites; the surplus electrolyte soaking towards the circular hole lithium anode during discharging; and the occurrence of a fast reaction front moving from the edge of the anode to the center of the cathode at the end of each discharge step (Figure 15).

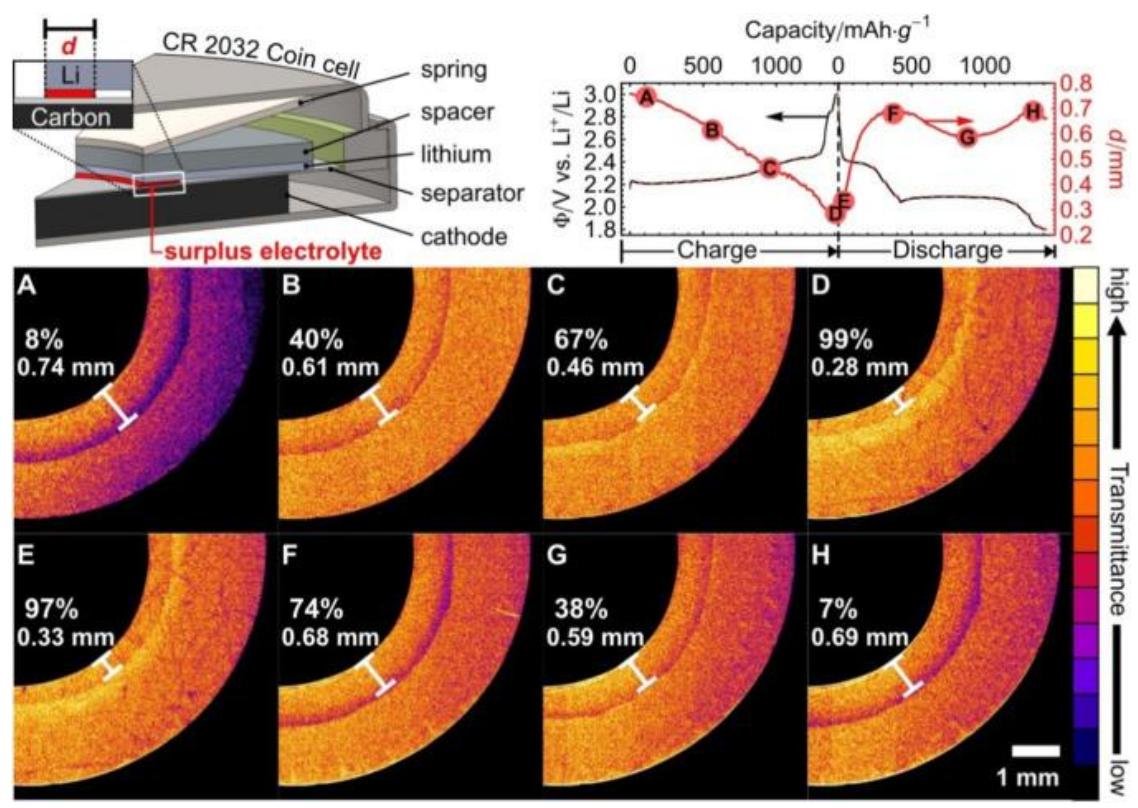

Figure 15. X-ray radiography images showing the evolution of surplus electrolyte ring between the separator and the lithium anode hole at different states $(A-H)$ of charge/discharge (\%) during the first cycle. The plot shows the charge/discharge curve and the measured thickness $d$ (red color in the scheme and the plot). Reproduced with permission from [107].

Suzer et al. reported the use of operando XPS for chemical and electrical measurement of a CdS-based light-dependent resistor, providing analyses of states under actual working conditions [108]. This work is not in the catalysis arena, but is very relevant to electrochemical changes. A particularly interesting technique is the scanning electrochemical microscopy (SCEM), an electroanalytical probe that scans substrate topography and local reactivity providing high resolution [109]. It has a major interest in the case of electrochemical reactions, as it was been reported by Fermin and coworkers, mapping hydrogen evolution reaction (HER) catalyzed by two-dimensional assemblies palladium nanoparticles on mica substrates [110]. They show that the potential of these nanoparticles is effectively determined by the local concentration ratio of the redox probe. The reactivity of the palladium nanoparticles is comparable to that of the bulk metal counterpart; this would be in line with the bulk-like electronic structure present in $\mathrm{Pd}$ nanoparticles of this size. 


\subsubsection{Mapping the fluid phase composition and states during operation}

Previous section points out how catalyst profiling/imaging is developing swiftly. However, the activity is still frequently measured at the end of pipe. Only few studies report spatially resolved kinetic data, and in these cases the characterization of the catalyst, with or without spatial resolution, is frequently missing.

Gaseous species profiles can be obtained with significantly high resolution by translation of one or more capillaries along the flow direction of a reactor and/or axial rotation. This method was developed independently at Oak Ridge National Lab (SpaciMS, marketed by Hiden Analytical) [62, 111], the Paul Scherrer Institute [112] and the University of Minnesota [113]. The analytical tool is usually mass spectrometry (MS), but a gas-phase FT-IR analyzer has also been used in a $\mathrm{NH}_{3}$-SCR monolithic catalyst [114], and gas chromatography (GC) could also be adapted [115]. Special attention must be paid to the alteration of the operating conditions by the probe, and time- or space-gaps between fluid and surface measurements should not be disregarded; these may introduce interpretation errors [111]. Capillary size MS probes minimally alter the flow regime in the system under study of Figure 16 [111], but they may have an effect on other configurations [63].

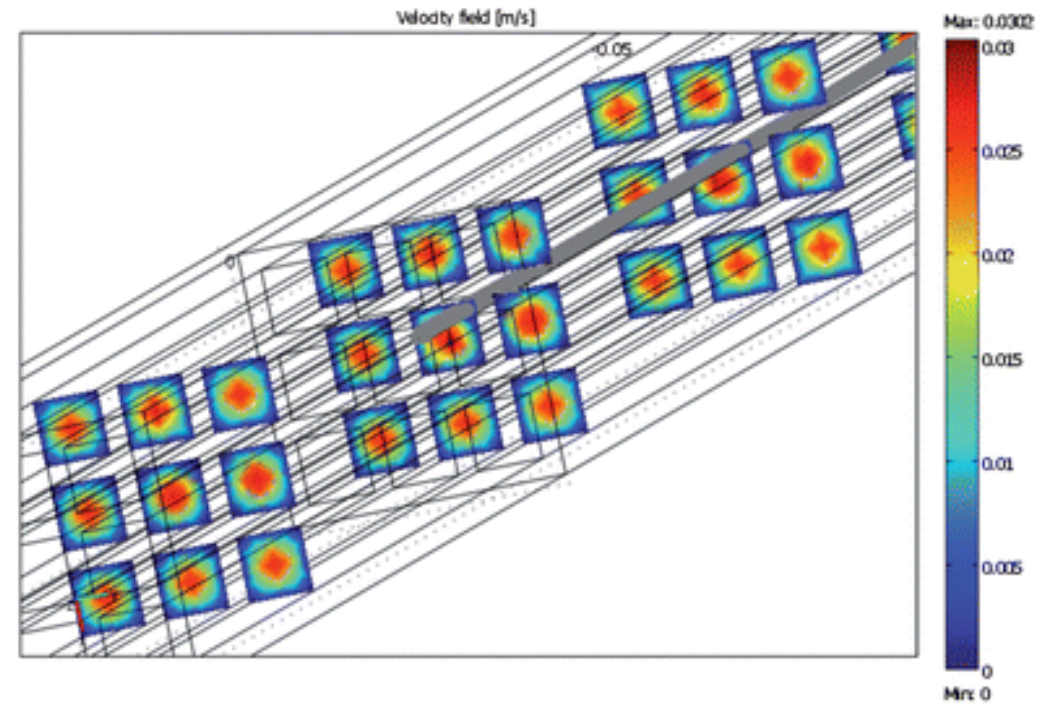

Figure 16. Calculated velocity profile in a monolith showing the negligible effect of a capillary MS probe situated in the corner of the central channel. $\mathrm{T}=200{ }^{\circ} \mathrm{C}, \mathrm{u}_{0}=0.016 \mathrm{~m} \mathrm{~s}^{-1}$, channel size $=0.001 \mathrm{~m}$, probe diameter $=250 \mu \mathrm{m}$. Reproduced with permission from [111]

Assessing what is the state of catalyst and reactants and how they both evolve not only in time, but also in space during reaction is still a challenge for the operando methodology. Simultaneous kinetic and spectroscopic characterization with spatial resolution in both activity and chemical structure measurements is rarely performed. Figure 17 illustrates this approach; in this case, temperature profiles are also obtained. Distortions caused by turbulence and backmixing produced at the capillary open end were avoided using a sealed capillary with a side sampling orifice [116]. The capillary was translated up and down with $\mu \mathrm{m}$ resolution through a fixed-bed tubular reactor by means of a stepper motor 
[117]. The spatial profiles of all major gas phase species of the oxidative dehydrogenation $(\mathrm{ODH})$ of ethane to ethylene were measured by MS through the sampling orifice, and gas temperature and the Raman shift on $\gamma-\mathrm{Al}_{2} \mathrm{O}_{3}$-supported $\mathrm{MoO}_{3}$ were monitored by a thermocouple or an optical fiber sensor, respectively, inserted into the capillary and aligned with the sampling orifice. It must be mentioned that the Raman fiber lacked sensitivity to follow all relevant vibrations and that the Raman spectra were corrected to remove the contribution from fused silica generated inside the fiber optic.



Figure 17. Simultaneous measurement of thermal, kinetic and spectroscopic profiles through a fixed-bed tubular reactor. Left: Catalytic bed of $50 \mathrm{wt} \% \quad \mathrm{MoO}_{3} / \mathrm{Y}$-alumina spheres, middle: probe geometry, right: Raman spectra, temperature and composition profiles. Reproduced with permission from [117].

All NMR spectroscopy methods can take advantage of MRI (magnetic resonance imaging); hence spatially resolved chemical conversion measurements are possible. However, obtaining adequate signal-to-noise ratios is a key requisite to achieve successful results, acquisition time may be long and NMR signal decreases with temperature. Several reviews provide a comprehensive view on operando applications of NMR in catalysis [118, 119]. The majority of the early operando NMR studies addressed the heterogeneity in transport within catalyst pellets. In-plane spatial resolution achieved in those investigations was approximately $30 \mu \mathrm{m}$, and the pellets themselves were of typical dimension $1-5 \mathrm{~mm}$. Conventional spin-echo imaging typically takes the order of a few minutes. NMR signal can be dramatically enhanced by hyperpolarization [120, 121]. Koptyug reported on high resolution applications of NMR in the study of microreactors [122]. The acquisition speed of an MR image may be improved by three sampling strategies: echo planar imaging (EPI), rapid acquisition with relaxation enhancement (RARE), and low excitation angle imaging [118].

An example of application of EPI is the study of a drying process depicted in Figure 18. This technique was sufficiently robust to spatially resolve drying within a packed bed of $100-\mu \mathrm{m}$ glass spheres. The motivation for this investigation was to understand the origins of heterogeneity in the optical properties of pigments dried from a slurry, but such data also provide insights into the effects of drying on the homogeneity of metal loading during catalyst manufacture. Although the drying process was characterized by a uniform water mass loss with time, the MR images clearly show the spatial heterogeneity in the drying 
process. These observations have been used to aid the development of an invasionpercolation modeling strategy, which predicts both the drying rate and the heterogeneity of the drying process [123].
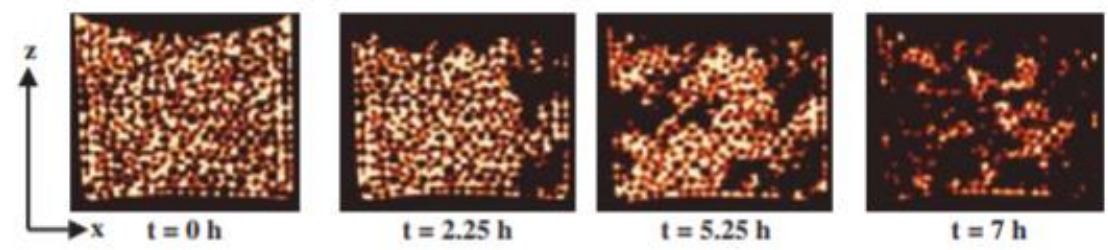

Figure 18. 2D slice section through 3D MR images of water distribution within an initially water-saturated packing of $500-\mu \mathrm{m}$ glass spheres. Voxel resolution is $94 \mathrm{~mm} \times 94 \mathrm{~mm} \times$ $94 \mathrm{~mm}$. Data are shown before drying commenced and at three time intervals during the drying process. Only the water within the inter-particle space of the bead packing was imaged (white pixels). No signal was obtained from the solid and gas phases present. Reproduced with permission from [123, 124].

Cattaneo et al. [125] reported the feasibility of operando ${ }^{1} \mathrm{H}$ electrochemical NMR microscopy for the determination of the proton distribution in membranes of polymer fuel cells at low humidity level. They studied the adsorbed water distribution before starting the cell operation and at operation. Their zero-time echo (ZTE) images (Figure 19) provide an insightful tool to investigate water production and management in fuel cells at high temperature and under low humidity conditions, which is relevant for automotive applications. Isotopic enrichment of the samples with ${ }^{2} \mathrm{H}$ provides means to investigate diffusion and exchange processes among the polymer matrix, phosphoric acid and water.
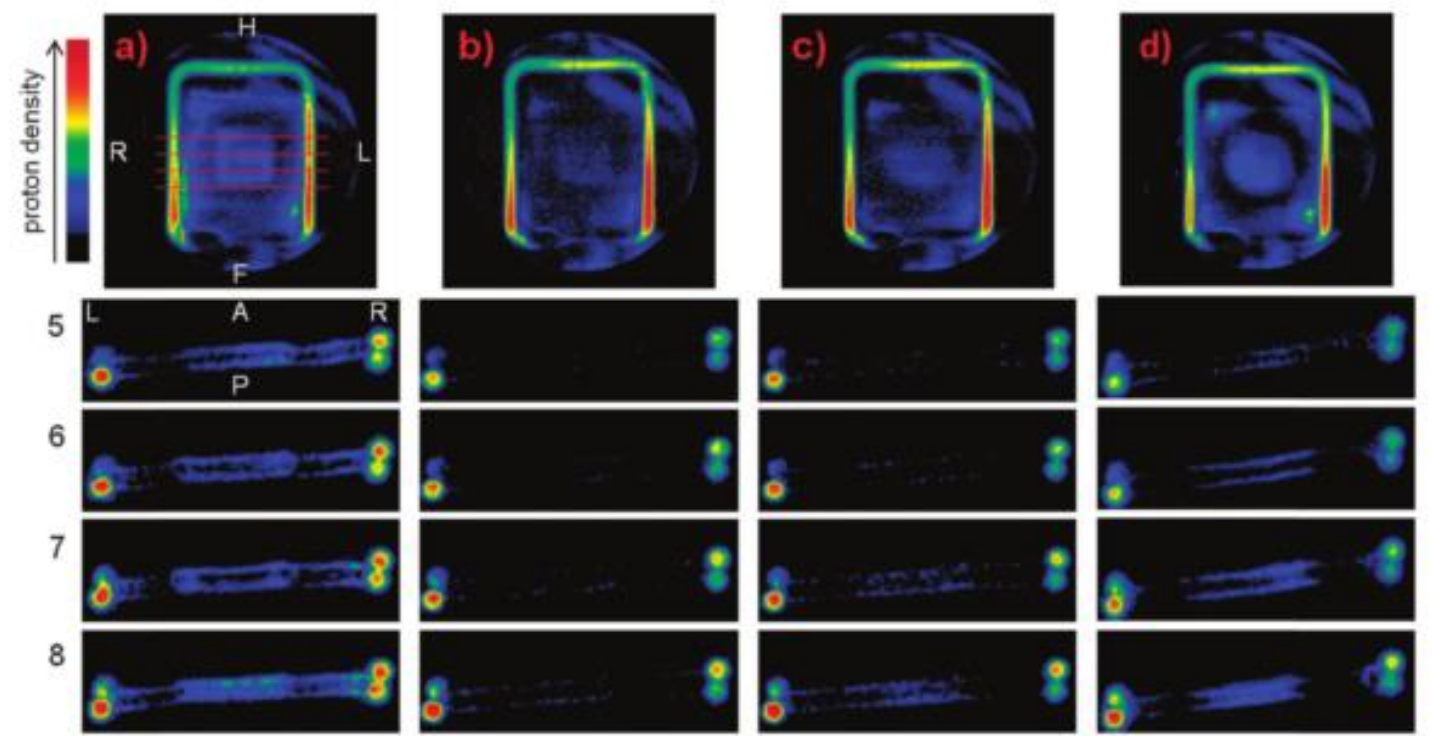

Figure 19. ${ }^{1} \mathrm{H}$ zero-time echo images and axial slices extracted from the corresponding 3D dataset obtained from a membrane-electrode assembly (MEA) operating with $\mathrm{H}_{2}$ and air at $80{ }^{\circ} \mathrm{C}$ and $30 \mathrm{~mA}$. (a) Fuel cell at $25^{\circ} \mathrm{C}$ before operation $(0 \mathrm{~V})$; (b) $\mathrm{t}=0(0.44 \mathrm{~V})$; (c) $\mathrm{t}=$ $420 \min (0.42 \mathrm{~V})$; (d) fuel cell at $25{ }^{\circ} \mathrm{C} 14$ hours after the switch off at $420 \mathrm{~min}$. Reproduced with permission from [125]. 
An additional advantage of magnetic resonance imaging is that it may analyze within visually opaque materials. RARE was applied, for example, to follow an oscillatory chemical reaction occurring within an opaque packed bed reactor [126]; MR offered the opportunity to map the chemical state of the heterogeneous flowing phase and the product distribution within it. The 2-D MR images of the traveling (reaction-diffusion) waves shown in Figure 20 were acquired in a tube of internal diameter $16 \mathrm{~mm}$ containing $1 \mathrm{~mm}$ glass beads filled with a manganese-catalyzed Belousov-Zhabotinsky solution. Vertical images from the center of the tube illustrate the displacement of the waves. Relaxation contrast was used to monitor in time and space the interchange of $\mathrm{Mn}^{2+}$ and $\mathrm{Mn}^{3+}$ ions in the stream.

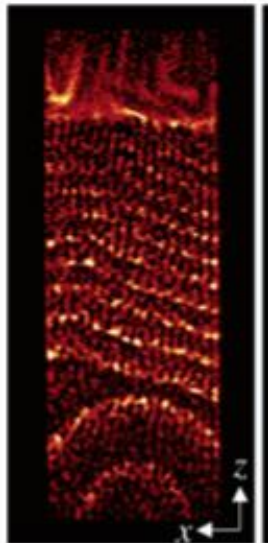

(a)

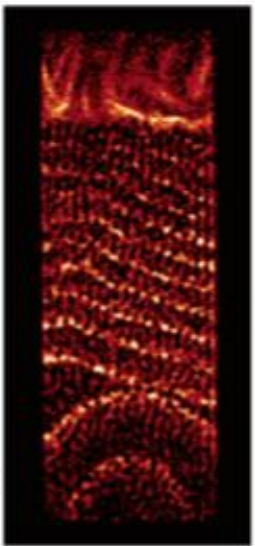

(b)

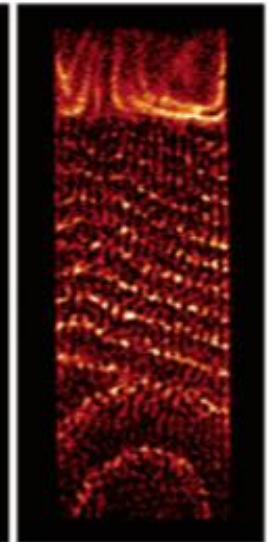

(c)

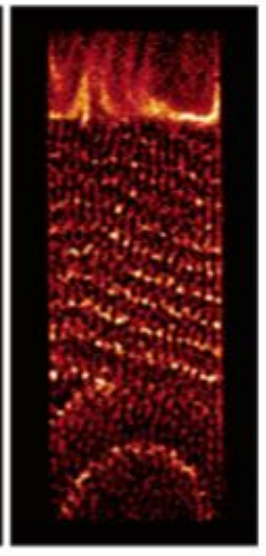

(d)

Figure 20. 2-D MR image of an oscillating chemical reaction occurring within a bed packed with glass beads. Chemical waves are imaged as a result of the oscillatory production of $\mathrm{Mn}^{2+}$ and $\mathrm{Mn}^{3+}$ species, identified as dark and light bands, respectively. Reproduced with permission from [126].

Ulpts et al. developed a 3D magnetic resonance spectroscopic imaging (MRSI) method to map the concentration distribution within regularly and irregularly structured monoliths [127]; it was validated with cordierite structures coated with $1 \mathrm{wt} \% \mathrm{Pt} / \mathrm{Al}_{2} \mathrm{O}_{3}$ during ethylene hydrogenation (Figure 21). With this method temperature can be mapped by using ethylene glycol filled glass capillaries [128].
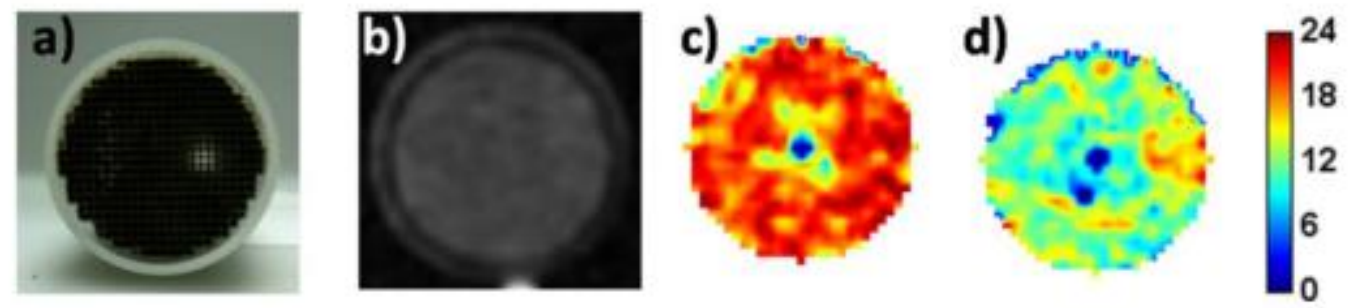


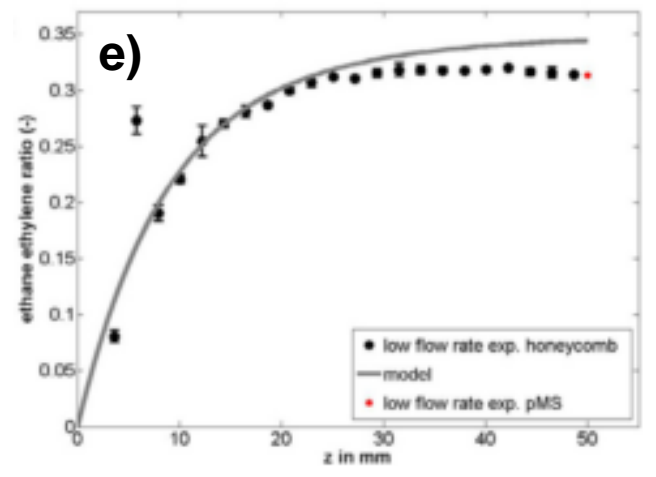

Figure 21. Operando study of ethylene hydrogenation over cordierite monoliths coated with $1 \%$ wt. $\mathrm{Pt} / \mathrm{Al}_{2} \mathrm{O}_{3}$ (a) Cross-section photographic image of the honeycomb catalyst, (b) NMR ethylene image of the cross section under a non-reactive mixture of ethylene and argon measured by 3D MRSI, (c) and (d) ethane concentration (\%vol) maps at low and high flow rate, respectively. e) Experimental and model ethane/ethylene ratio profile of the low flow-rate experiment. Adapted from [127].

Zheng et al. developed a method to calculate liquid-solid mass transfer coefficients and map inter- and intra-pellet chemical composition within fixed-bed reactors by spaceresolved ${ }^{13} \mathrm{C}$ Distortionless Enhancement by Polarisation Transfer $\left({ }^{13} \mathrm{C}\right.$ DEPT) pulse sequence NMR; the partial least squares regression method was used to discriminate between intra- and inter-pellet species [129]. This allowed them to detect changes in selectivity along the reactor during 1-octene hydrogenation and product accumulation inside the $0.3 \mathrm{wt} \% \% \mathrm{Pd} / \mathrm{Al}_{2} \mathrm{O}_{3}$ catalyst, attributed to mass transfer limitations. They calculated the bed porosity/wetting efficiency/total liquid holdup from the ratio between the number of pixels associated with the void space/wetted surface/interpellet liquid and the cross-section of the bed with Figure 22 images.
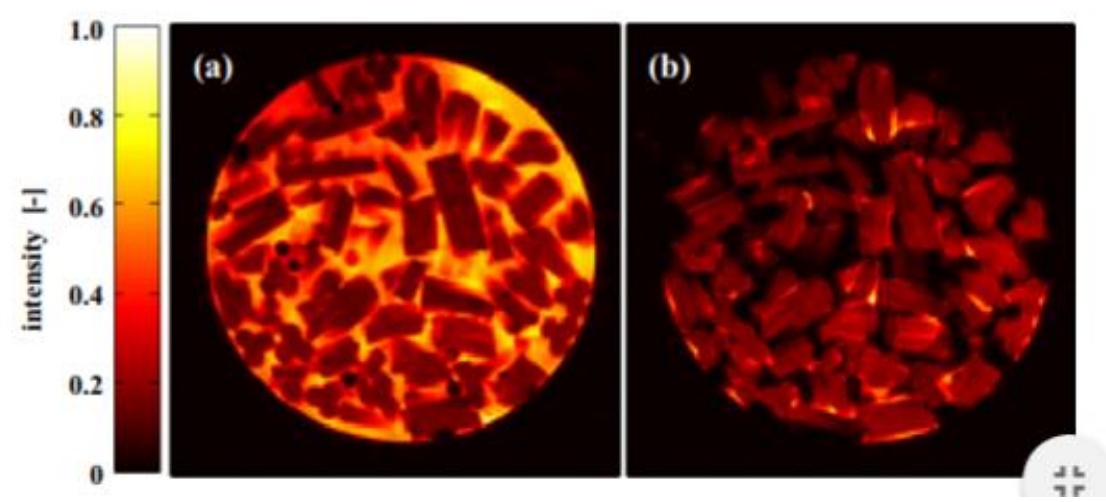

Figure 22. 2D ${ }^{1} \mathrm{H} \mathrm{MRI}$ data from which bed porosity, liquid holdup and wetting efficiency of $0.3 \mathrm{wt} \% \mathrm{Pd} / \mathrm{Al}_{2} \mathrm{O}_{3}$ catalyst pellets were calculated. (a) bed flooded with liquid 1-octene, and (b) bed during 1-octene hydrogenation reaction. Reproduced with permission from [129].

\subsubsection{Temperature mapping in operando studies}


Temperature profiles can be measured with thermocouples/temperature sensors inside moving capillaries. This option is invasive, and thus can alter local heat and mass transport properties [130], but it is an easy solution to complement MS capillary profiles (see Figure 17). Thermocouples focus mainly on the gas phase, and not on the interphase, while infrared thermography can monitor the solid surface temperature profiles. In a pioneering work, Wolf and coworkers [131-133] reported the use of infrared thermography to monitor transient and oscillatory behaviors of several catalysts for $\mathrm{CO}$ oxidation (Figure 23); those oscillations were connected to changes on the surface state of the catalyst [131].
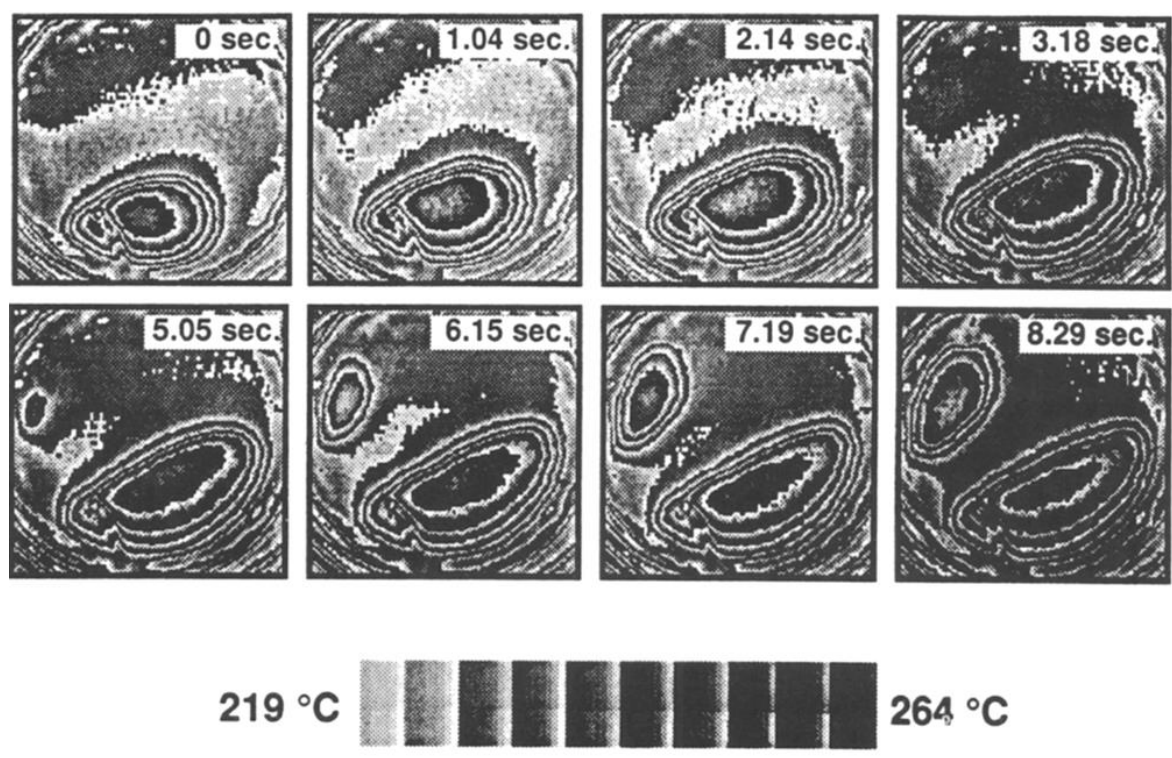

Figure 23. Thermographs showing an ignition sequence on a $5 \mathrm{wt} . \% \mathrm{Rh} / \mathrm{SiO}_{2}$ catalyst wafer; the flow is parallel to the wafer surface, flowing from the bottom left corner to the top right one. Reproduced with permission [132, 133].

The combination of temperature profiling by spatially resolved NMR thermometry with activity measurements unveils close relationships between catalytic performance and catalyst state, albeit without chemical characterization [134, 135]. Deeper insight is enabled when chemical structure characterization is available. Infrared thermography was used in combination with a high-speed X-ray camera and online reactor effluent analysis by mass spectrometry, which was reported for the ignition of the catalytic partial oxidation of methane in a fixed-bed capillary microreactor, as shown in Figure 24 [136]. The authors could show how catalyst reduction and reaction ignition are deeply entangled. The temperature profiles vs. time in Figure $24 \mathrm{f}$ are from a separate experiment in the same cell, since X-ray absorption configuration does not allow simultaneous profiling of temperature. 


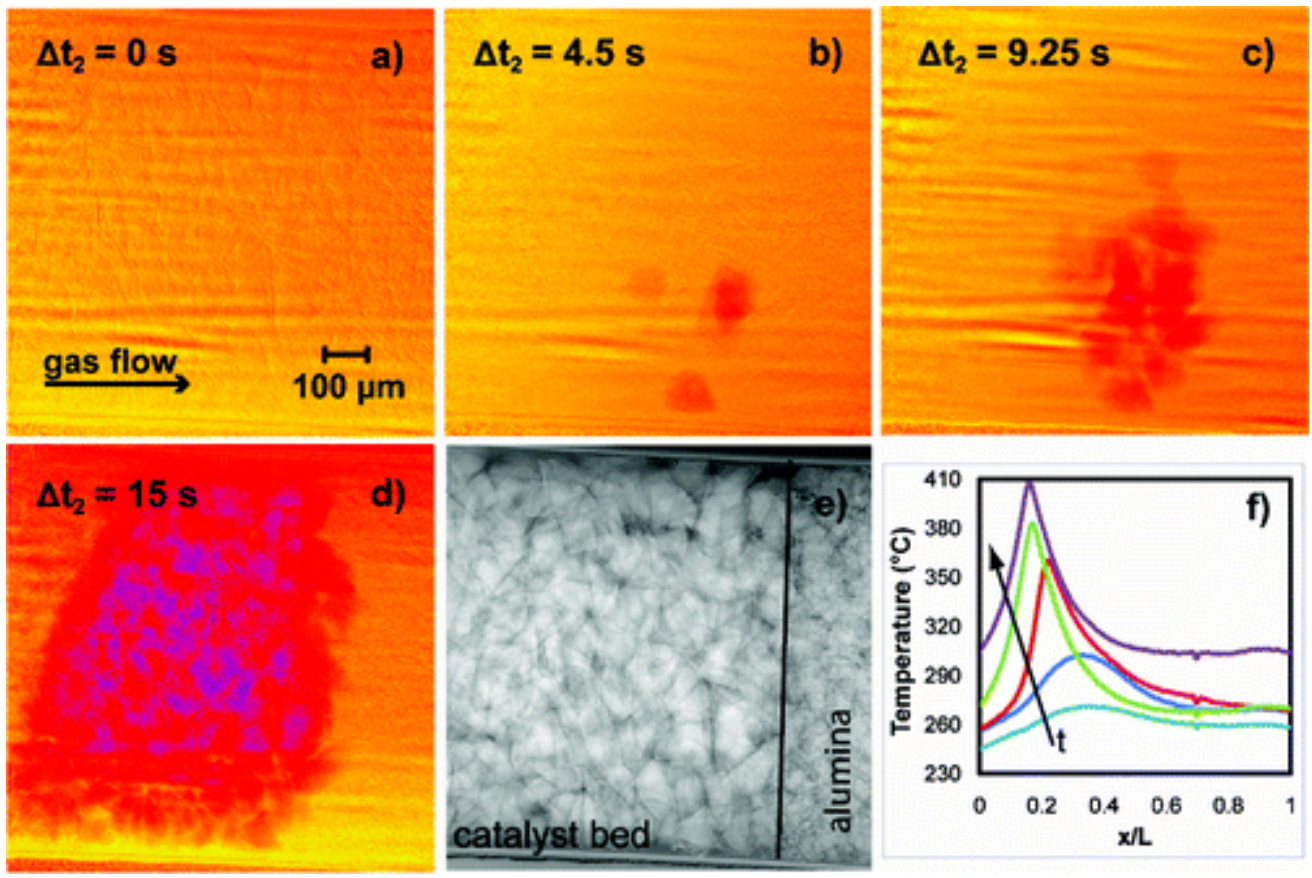

Figure 24. Space-resolved operando study on the ignition of the catalytic partial oxidation of methane in a fixed-bed capillary microreactor. (a)-(d) Oxidized catalyst (yellow-orange), formation of the front of reduction (red-violet), advance of the front towards the inlet. (e) Xray absorption image where the single reduced particles can be identified (f) Temperature profile evolution measured by IR-thermography. Reproduced with permission from [136].

\subsection{Operando studies in microstructured reactors}

Many studies have been carried out combining different spectroscopic techniques with microfluidic reactors, but operando studies relevant to heterogeneous catalysis are rather limited, despite the interest of this kind of reactors owing to the advantages mentioned in Section 1.

An early work was an operando Raman-GC study reported by Cao et al. [137] on methanol oxidation over silver in a silicon-glass microreactor. The authors analyzed local microstructural changes by single-point Raman during oxidation/reaction activation cycles and their influence on the activity and selectivity for the catalytic oxidation of methanol to formaldehyde. These studies allowed to: (i) identify the stabilization of subsurface atomic oxygen species as determinant to obtain higher $\mathrm{CH}_{2} \mathrm{O}$ yield, (ii) detect the silver surface restructuration by methanol oxidation (Figure 25). 


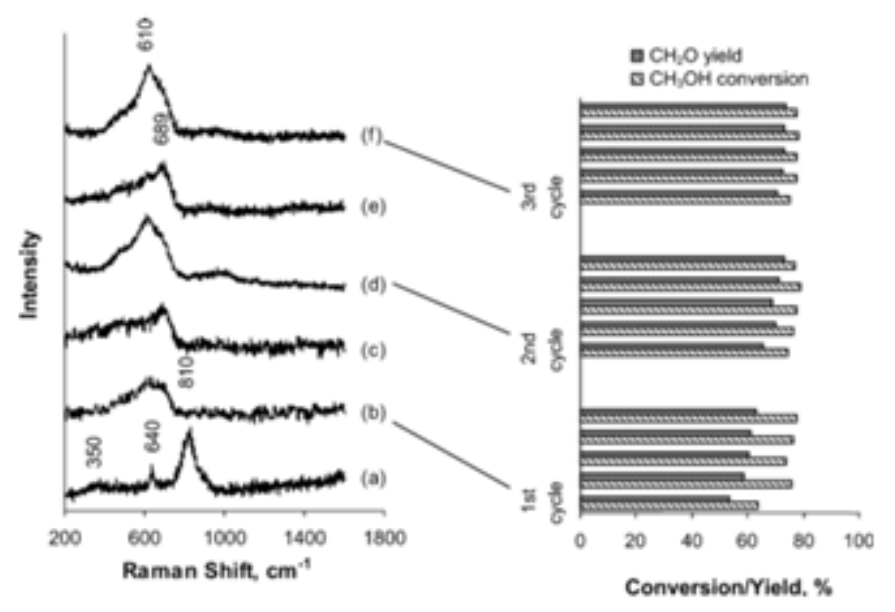

Figure 25. Operando Raman-GC results of silver catalyst during oxidation/reaction cycles at $773 \mathrm{~K}$. Raman spectra at the end of: (a, c, e) oxidation, and (b, d, f) reaction steps. Oxidation in a flow of $\mathrm{O}_{2}(4.1 \%)$, He as balance; reaction in a flow of $\mathrm{CH}_{3} \mathrm{OH}(8.75 \%), \mathrm{O}_{2}$ $(3.5 \%)$ and $\mathrm{H}_{2} \mathrm{O}(6.63 \%)$, He as balance. Reproduced with permission from [137].

Some relevant space-resolved studies using capillary reactors have been already commented in previous sections (see Figure 12 and Figure 24). In another capillary microreactor, loaded with $\mathrm{Pt} / \mathrm{Al}_{2} \mathrm{O}_{3}$, Gänzler et al. [138] combined global online catalytic activity measurements with spatially and time-resolved IR thermography and XANES/EXAFS to investigate the oscillatory CO oxidation. This combination allowed them to correlate the catalytic performance with local thermal effects and structural changes. The results showed a cyclic switch between more active reduced Pt particles and less active oxidized platinum (Figure 26), bringing understanding on parameters connected with the oscillatory $\mathrm{CO}$ conversion. The position of the active and inactive catalyst zones could be distinguished and was identified to depend on the reaction conditions. 


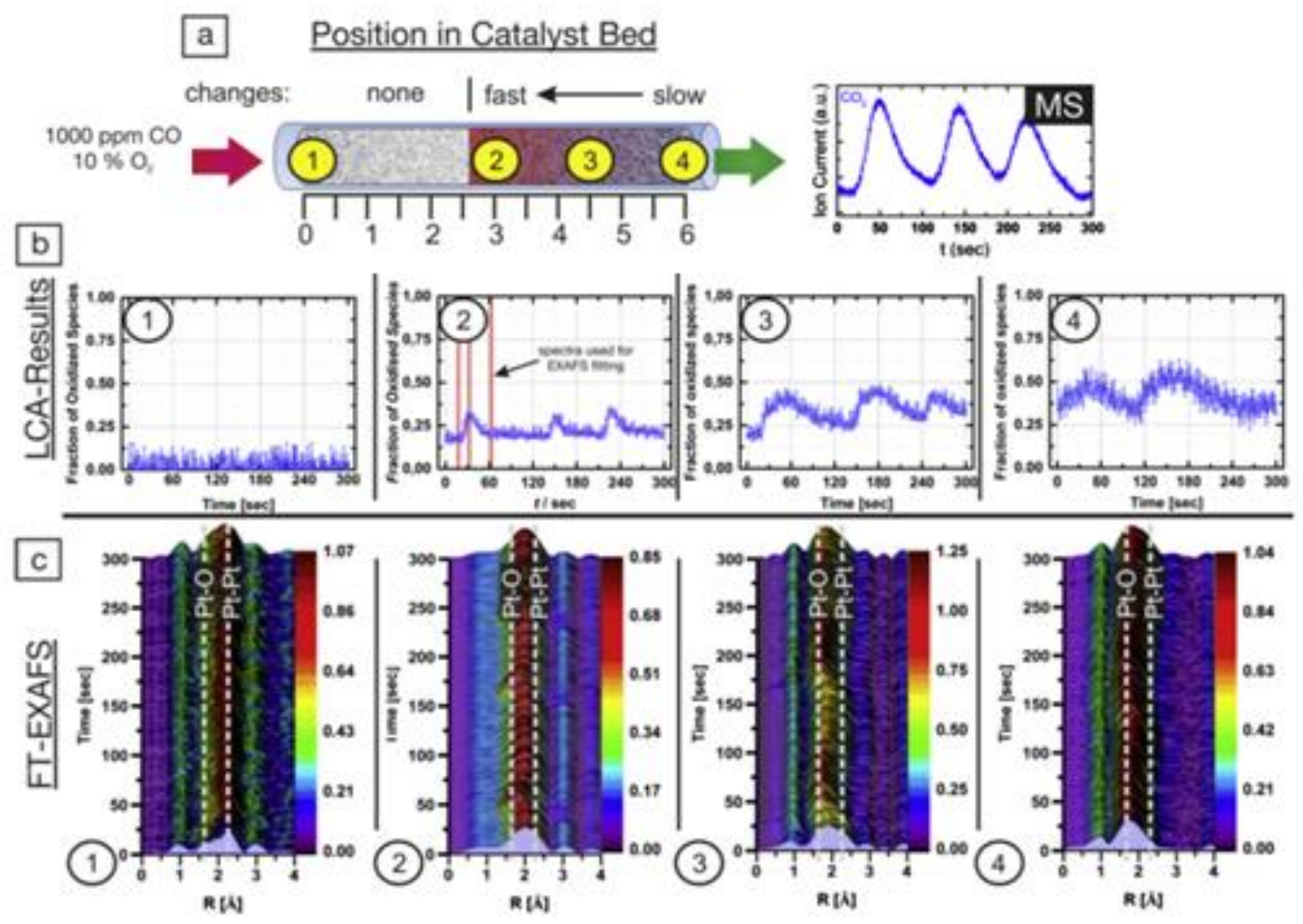

Figure 26. Space- and time-resolved data during $\mathrm{CO}$ oxidation at $110{ }^{\circ} \mathrm{C}$ with a Pt catalyst in a capillary reactor in $1000 \mathrm{ppm} \mathrm{CO}, 10 \% \mathrm{O}_{2}$ in $\mathrm{He}$, total flow of $50 \mathrm{~mL} \cdot \mathrm{min}^{-1}$. (a) Catalyst bed scheme with the characterization points location and global gas-phase MS results. (b) LCF of XANES spectra. (c) k2-weighted Fourier-transformed QEXAFS data (k-range: 3.0$9.0 \AA^{-1}$ ). Reproduced with permission form [138].

Grunwaldt and coworkers mapped chemical structure heterogeneities of $\mathrm{Rh} / \mathrm{Al}_{2} \mathrm{O}_{3}$ [139] and $\mathrm{Rh}-\mathrm{Pt} / \mathrm{Al}_{2} \mathrm{O}_{3}$ [140] catalyst in a capillary bed by XAS during the catalytic partial oxidation of methane, while following the global reaction by on-line mass spectrometry. The rhodium and platinum species were mainly in oxidized states in the entrance zone of the catalyst bed, whereas more reduced noble metal species were detected downstream (Figure 27). Temperature and space velocity were found to determine the relative distribution of Rh-species, as expected by their influence on the relative distribution of the reaction products.
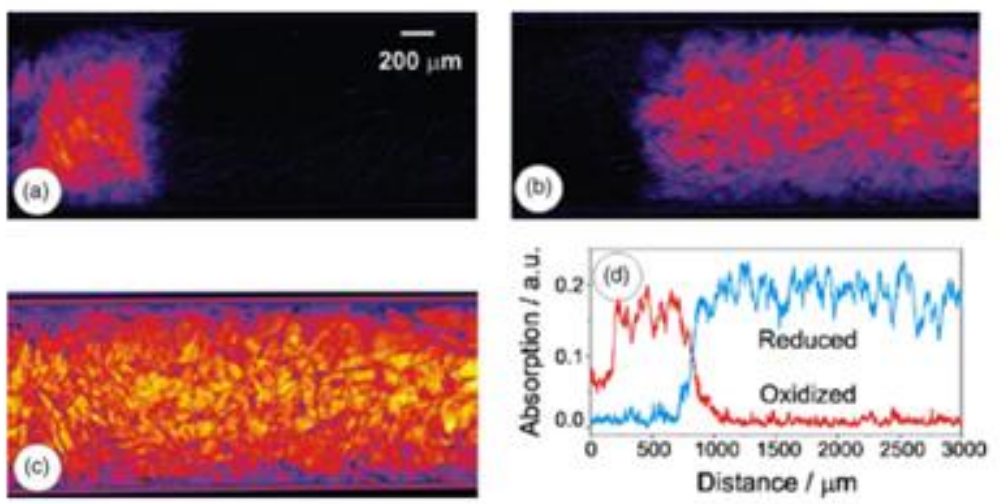
Figure 27. Map of a $\mathrm{Rh} / \mathrm{Al}_{2} \mathrm{O}_{3}$ catalyst during methane partial oxidation. (a) Oxidized Rhspecies; (b) reduced Rh-species; (c) featureless background; (d) relative concentration of the oxidized and reduced Rh-particles in the axis of the fixed-bed (conditions: $\mathrm{T}=362{ }^{\circ} \mathrm{C}$, space velocity $\left.=1.9 \times 10^{5} \mathrm{~h}^{-1}\right)$. Reproduced with permission from [139].

In microreactors, as in macroreactors, chemical profiles occur in the catalyst, but also in the fluid phase; the latter heterogeneity is illustrated for example by Fletcher et al., using an inverted Raman microscope spectrometer to profile the spatial evolution of reactant and product concentrations within a T-shaped channel network [141]. The synthesis of ethyl acetate from ethanol and acetic acid was investigated as a model system (Figure 28). The contact between the two liquids and the formation of co-flowing laminar streams down the leg of the "T" can be clearly seen. Under steady-state conditions the concentration at each position corresponds to a different time on stream.
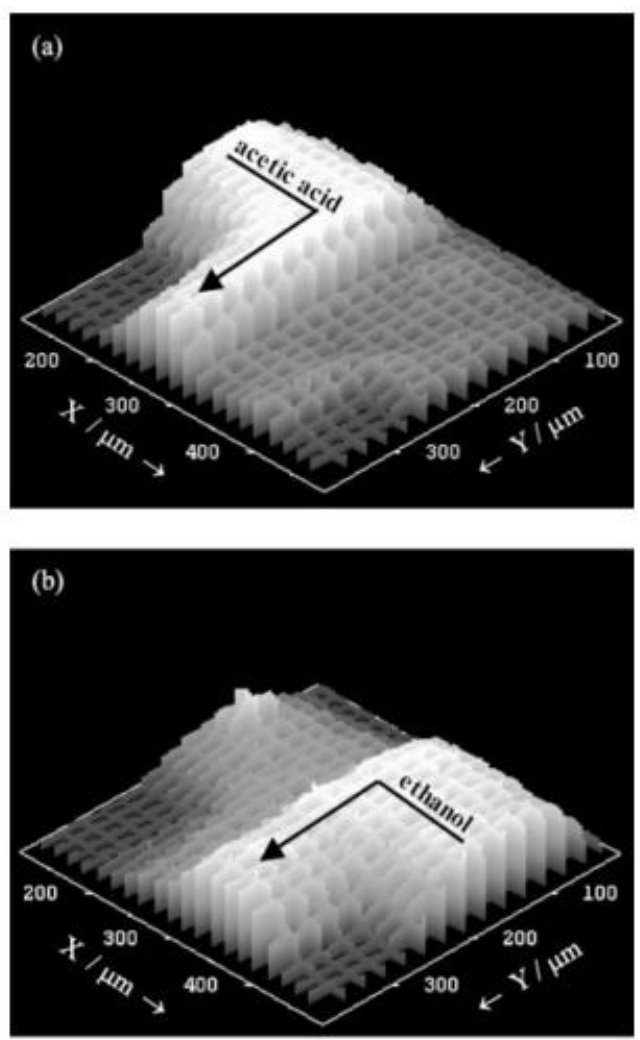

Figure 28. 3D plots of Raman intensity in the T-junction region for specific bands: (a) 893 $\mathrm{cm}^{-1}$ from acetic acid, and (b) $882 \mathrm{~cm}^{-1}$ from ethanol. Reproduced with permission from [141].

These works illustrate the state of the art in space-resolved monitoring the nature of the catalyst at work and the state of the reaction in microreactors; both views, of the catalyst and the reaction, are the pillars of operando methodology, blending these approaches in one experiment will deliver the much-needed complete spatial resolution to operando studies. 


\section{Concluding Outlook: measure locally, think globally}

Operando methodology appeared as an answer to the need of characterizing chemical structure and activity in a simultaneous manner. This approach provides the best positioning to assess structure-activity relationships at a molecular scale and has been very successful. Deeper knowledge of catalysis through operando methodology has multifold evolutions. One is that complementary insights became important: several groups developed multispectroscopic operando set-ups so that information about the catalyst came from several techniques; this has been instrumental for major progress in understanding catalysis. Another evolution arises from the fact that single-point local operando spectroscopy misses a reality that occurs at laboratory-scale reactors and that is determining in larger industrial reactors: the existence of gradients. Catalysis is a phenomenon that takes place locally, but it has to be understood globally, because catalytic processes progress in time and in space. The state of the active sites at a given time is not the same at several locations of the catalytic reactor. As we move downstream the catalytic bed the reactants are increasingly converted, generating reaction intermediates and products. Gradients of temperature, fluid phase composition, adsorbatecatalyst interaction, and the very state of the catalyst are immediate consequences. A molecularly local insight into the catalytic act hampers a global understanding of the process. Connecting global activity of a catalyst bed with its structure at specific locations has uncertainties that can only be minimized operating in a differential regime. This approach is valid for fundamental research, delivering molecular comprehension of structure-activity relationships, but it is increasingly hampered as we mimic more real systems, where conversion usually goes beyond differential regime values.

Spatial evolution over time of temperature (of fluid and solid phases), hydrodynamics and chemistry along the reactor must be known as well, which requires that operando methodologies become space-resolved not only regarding the spectroscopic characterization, but also the activity measurement and other variables relevant to the catalytic act. Mapping both the catalyst state and reactivity will provide the ultimate tool to scaling-up the operando methodology to real catalytic systems.

Several approaches face the challenges of time-resolved in situ mapping of catalysts at work and of time-resolved reaction imaging. This review highlights how these are poised to blend into time-resolved operando mapping of catalysts and catalysis. The successful implementation of space- and time-resolved operando methodology not only paves the ground to understand real industrial catalysts, but may also provide feedback for real-time control of the catalytic process and optimization of the catalyst performance and stability.

\section{Acknowledgements}


This work was supported by Spanish Ministry grants CTQ2014-57578-R "LT-NOx" and CTM2017-82335-R „RIEN 2 O“; and Comunidad de Madrid progamme S2013/MAE-2985 "ALCCONES". 


\section{References}

1. Mitchell S, Michels N-L, Perez-Ramirez J. From powder to technical body: the undervalued science of catalyst scale up. Chemical Society Reviews, 2013, 42(14): 60946112

2. Boger T, Heibel A K, Sorensen C M. Monolithic catalysts for the chemical industry. Industrial \& Engineering Chemistry Research, 2004, 43(16): 4602-4611

3. Scheffler F, Claus P, Schimpf S, Lucas M, Scheffler M. Heterogeneously catalyzed processes with porous cellular ceramic monoliths. Cellular ceramics: Wiley-VCH Verlag GmbH \& Co. KGaA, 2006, 454-483

4. Tronconi E, Groppi G, Visconti C G. Structured catalysts for non-adiabatic applications. Current Opinion in Chemical Engineering, 2014, 5: 55-67

5. Kreutzer M T, Kapteijn F, Moulijn J A, Heiszwolf J J. Multiphase monolith reactors: Chemical reaction engineering of segmented flow in microchannels. Chemical Engineering Science, 2005, 60(22): 5895-5916

6. Twigg M V, Richardson J T. Fundamentals and applications of structured ceramic foam catalysts. Industrial \& Engineering Chemistry Research, 2007, 46(12): 4166-4177

7. Jähnisch K, Hessel V, Löwe H, Baerns M. Chemistry in microstructured reactors. Angewandte Chemie International Edition, 2004, 43(4): 406-446

8. Yue J, Schouten J C, Nijhuis T A. Integration of microreactors with spectroscopic detection for online reaction monitoring and catalyst characterization. Industrial \& Engineering Chemistry Research, 2012, 51(45): 14583-14609

9. Kestenbaum H, Lange de Oliveira A, Schmidt W, Schüth F, Ehrfeld W, Gebauer K, Löwe H, Richter T, Lebiedz D, Untiedt I, Züchner H. Silver-catalyzed oxidation of ethylene to ethylene oxide in a microreaction system. Industrial \& Engineering Chemistry Research, 2002, 41(4): 710-719

10. Inoue T, Schmidt M A, Jensen K F. Microfabricated multiphase reactors for the direct synthesis of hydrogen peroxide from hydrogen and oxygen. Industrial \& Engineering Chemistry Research, 2007, 46(4): 1153-1160

11. Yoshida J, Nagaki A, Iwasaki T, Suga S. Enhancement of chemical selectivity by microreactors. Chemical Engineering \& Technology, 2005, 28(3): 259-266

12. Al-Rifai N, Cao E, Dua V, Gavriilidis A. Microreaction technology aided catalytic process design. Current Opinion in Chemical Engineering, 2013, 2(3): 338-345

13. Ufer A, Sudhoff D, Mescher A, Agar D W. Suspension catalysis in a liquid-liquid capillary microreactor. Chemical Engineering Journal, 2011, 167(2): 468-474

14. Martin A J, Mitchell S, Kunze K, Weston K C, Pérez-Ramírez J. Visualising compositional heterogeneity during the scale up of multicomponent zeolite bodies. Materials Horizons, 2017, 4(5): 857-861

15. Rasmussen S B, López-Medina R, Portela R, Mikolajska E, Daturi M, Avila P, Bañares M A. Shaping up operando spectroscopy: Raman characterization of a working honeycomb monolith. Catalysis Science \& Technology, 2015, 5(11): 4942-4945 
16. Hunger M. W J. In situ IR, NMR, EPR, and UV/Vis spectroscopy: tools for new insight into the mechanisms of heterogeneous catalysis. Angewandte Chemie International Edition, 2001, 40: 2954-2971

17. Urakawa A. Trends and advances in operando methodology. Current Opinion in Chemical Engineering, 2016, 12: 31-36

18. Urakawa A, Maeda N, Baiker A. Space- and time-resolved combined DRIFT and Raman spectroscopy: Monitoring dynamic surface and bulk processes during NOx storage reduction. Angewandte Chemie International Edition, 2008, 47(48): 9256-9259

19. Burch R. In situ methods in catalysis-Proceedings of the Surface Reactivity and Catalysis Group Meeting of the Royal Society of Chemistry. Catalysis Today, 1991, 9(1-2)

20. Clausen B S, Topsøe H, Frahm R. Application of combined X-ray diffraction and absorption techniques for in situ catalyst characterization. In: Eley D D, Haag W O, Gates B, Knözinger H, (eds). Advances in Catalysis: Academic Press, 1998, bu315-344

21. Dumesic J A, Topsøe H. Mössbauer Spectroscopy Applications to Heterogeneous Catalysis. In: Eley D D, Pines H, Weisz P B, (eds). Advances in Catalysis: Academic Press, 1977, 121-246

22. Bañares M A. Operando spectroscopy: The knowledge bridge to assessing structure-performance relationships in catalyst nanoparticles. Advanced Materials, 2011, 23(44): 5293-5301

23. Topsøe H. Developments in operando studies and in situ characterization of heterogeneous catalysts. Journal of Catalysis, 2003, 216(1-2): 155-164

24. Topsøe H. In situ characterization of catalysts. In: Corma A, Melo F V, Mendioroz S, Fierro J L G, (eds). 12th International Congress on Catalysis, Proceedings of the 12th ICC: Elsevier, 2000, 1-21

25. Meunier F C. The design and testing of kinetically-appropriate operando spectroscopic cells for investigating heterogeneous catalytic reactions. Chemical Society Reviews, 2010, 39(12): 4602-4614

26. Weckhuysen B M. Preface: recent advances in the in-situ characterization of heterogeneous catalysts. Chemical Society Reviews, 2010, 39(12): 4557-4559

27. Weckhuysen B M. Snapshots of a working catalyst: possibilities and limitations of in situ spectroscopy in the field of heterogeneous catalysis. Chemical Communications, 2002, 2: $97-110$

28. Bañares M A, Guerrero-Pérez M O, Fierro J L G, Garcia Cortez G. Raman spectroscopy during catalytic operations with on-line activity measurement (operando spectroscopy): A method for understanding the active centres of cations supported on porous materials. Journal of Materials Chemistry, 2002, 12(11): 3337-3342

29. Calvino-Casilda V, Banares M A. Recent advances in imaging and monitoring of heterogeneous catalysts with Raman spectroscopy. Catalysis: Volume 24: The Royal Society of Chemistry, 2012, 1-47

30. Banares M A. In situ to operando spectroscopy: From proof of concept to industrial application. Topics in Catalysis, 2009, 52(10): 1301-1302

31. Niemantsverdriet J W. Spectroscopy in catalysis: an introduction, 3rd, completely revised and enlarged edition: Wiley, 2007

32. Somorjai G A. In situ surface science studies of catalytic reactions. CATTECH, 1999, 3(1): 84-97

33. Bennici S M, Vogelaar B M, Nijhuis T A, Weckhuysen B M. Real-time control of a catalytic solid in a fixed-bed reactor based on in situ spectroscopy. Angewandte Chemie International Edition, 2007, 46(28): 5412-5416

34. Brückner A, Kondratenko E. Simultaneous operando EPR/UV-vis/laser-Raman spectroscopy - A powerful tool for monitoring transition metal oxide catalysts during reaction. Catalysis Today, 2006, 113(1-2): 16-24 
35. Bañares M A, Mestl G. Structural characterization of catalysts by operando Raman spectroscopy. In-situ Characterization of Heterogeneous Catalysts, 2013, 267-292 36. Balboni M L. Process analytical technology: Concepts and principles. Pharmaceutical Technology, 2003, 27(10): 54

37. Vogt C, Weckhuysen B M, Ruiz-Martínez J. Effect of feedstock and catalyst impurities on the methanol-to-olefin reaction overH-SAPO-34. ChemCatChem, 2017, 9(1): 183-194

38. Jentoft F C. Chapter 3 Ultraviolet-visible-near infrared spectroscopy in catalysis: theory, experiment, analysis, and application under reaction conditions. Advances in Catalysis, 2009, 52: 129-211

39. Rasmussen S B, Banares M A, Bazin P, Due-Hansen J, Avila P, Daturi M. Monitoring catalysts at work in their final form: spectroscopic investigations on a monolithic catalyst. Physical Chemistry Chemical Physics, 2012, 14(7): 2171-2177

40. $\quad$ Ferraro J R, Nakamoto K, Brown C W. Introductory Raman spectroscopy. In: Nakamoto K, Brown C W, (eds). Introductory Raman spectroscopy (Second Edition). San Diego: Academic Press, 2003, 1-434

41. Brückner A. Electron paramagnetic resonance: a powerful tool for monitoring working catalysts. Advances in Catalysis, 2007, 51: 265-308

42. Ivanova I I, Kolyagin Y G. Impact of in situ MAS NMR techniques to the understanding of the mechanisms of zeolite catalyzed reactions. Chemical Society Reviews, 2010, 39(12): 5018-5050

43. Blasco T. Insights into reaction mechanisms in heterogeneous catalysis revealed by in situ NMR spectroscopy. Chemical Society Reviews, 2010, 39(12): 4685-4702

44. Beale A M, Jacques S D M, Weckhuysen B M. Chemical imaging of catalytic solids with synchrotron radiation. Chemical Society Reviews, 2010, 39(12): 4656-4672

45. Newton M A, van Beek W. Combining synchrotron-based X-ray techniques with vibrational spectroscopies for the in situ study of heterogeneous catalysts: a view from a bridge. Chemical Society Reviews, 2010, 39(12): 4845-4863

46. Senyshyn A, Mühlbauer M J, Nikolowski K, Pirling T, Ehrenberg H. "In-operando" neutron scattering studies on Li-ion batteries. Journal of Power Sources, 2012, 203: 126129

47. Lennon D, Parker S F. Inelastic neutron scattering studies of methyl chloride synthesis over alumina. Accounts of Chemical Research, 2014, 47(4): 1220-1227

48. $\quad$ Frenken J, Groot I. Operando Research in Heterogeneous Catalysis: Springer International Publishing, 2017

49. Han B, Stoerzinger K A, Tileli V, Gamalski A D, Stach E A, Shao-Horn Y. Nanoscale structural oscillations in perovskite oxides induced by oxygen evolution. Nature Materials, 2017, 16(1): 121-126

50. Stavitski E, Weckhuysen B M. Infrared and Raman imaging of heterogeneous catalysts. Chemical Society Reviews, 2010, 39(12): 4615-4625

51. Weckhuysen B M. Chemical imaging of spatial heterogeneities in catalytic solids at different length and time scales. Angewandte Chemie - International Edition, 2009, 48(27): 4910-4943

52. Buurmans I L C, Weckhuysen B M. Heterogeneities of individual catalyst particles in space and time as monitored by spectroscopy. Nature Chemistry, 2012, 4(11): 873-886

53. Morgan K, Touitou J, Choi J-S, Coney C, Hardacre C, Pihl J A, Stere C E, Kim M-Y, Stewart C, Goguet A, Partridge W P. Evolution and enabling capabilities of spatially resolved techniques for the characterization of heterogeneously catalyzed reactions. ACS Catalysis, 2016, 6(2): 1356-1381 
54. Sattler J J H B, Mens A M, Weckhuysen B M. Real-time quantitative operando raman spectroscopy of a $\mathrm{CrOx} / \mathrm{Al} 2 \mathrm{O} 3$ propane dehydrogenation catalyst in a pilot-scale reactor. ChemCatChem, 2014, 6(11): 3139-3145

55. Guerrero-Pérez M O, Bañares M A. Operando raman study of alumina-supported $\mathrm{Sb}-\mathrm{V}-\mathrm{O}$ catalyst during propane ammoxidation to acrylonitrile with on-line activity measurement. Chemical Communications, 2002, 12: 1292-1293

56. Bañares M A, Wachs I E. Molecular structures of supported metal oxide catalysts under different environments. Journal of Raman Spectroscopy, 2002, 33(5): 359-380

57. Wachs I E. International Congress on Operando Spectroscopy: Fundamental and technical aspects of spectroscopy of catalysts under working conditions Lunteren, The Netherlands (March 2-6, 2003). Catalysis Communications, 2003, 4(11): 567-570

58. $\quad$ Chakrabarti A, Ford M E, Gregory D, Hu R, Keturakis C J, Lwin S, Tang Y, Yang Z, Zhu M, Bañares M A, Wachs I E. A decade+ of operando spectroscopy studies. Catalysis Today, 2017, 283: 27-53

59. Thomas S, Marie O, Bazin P, Lietti L, Visconti C G, Corbetta M, Manenti F, Daturi M. Modelling a reactor cell for operando IR studies: From qualitative to fully quantitative kinetic investigations. Catalysis Today, 2017, 283: 176-184

60. Thibault-Starzyk F, Seguin E, Thomas S, Daturi M, Arnolds H, King D A. Realtime infrared detection of cyanide flip on silver-alumina $\mathrm{NO} x$ removal catalyst. Science, 2009, 324(5930): 1048-1051

61. Krivanek O L, Lovejoy T C, Dellby N, Aoki T, Carpenter R W, Rez P, Soignard E, Zhu J, Batson P E, Lagos M J, Egerton R F, Crozier P A. Vibrational spectroscopy in the electron microscope. Nature, 2014, 514(7521): 209-212

62. Choi J S, Partridge W P, Daw C S. Spatially resolved in situ measurements of transient species breakthrough during cyclic, low-temperature regeneration of a monolithic $\mathrm{Pt} / \mathrm{K} / \mathrm{Al} 2 \mathrm{O} 3$ NOx storage-reduction catalyst. Applied Catalysis A: General, 2005, 293(1-2): 24-40

63. Nguyen H, Peng P Y, Luss D, Harold M P. Assessing intrusion by the capillary during spatially resolved mass spectrometry measurement. Chemical Engineering Journal, 2017, 307: 845-859

64. Bentrup U. Combining in situ characterization methods in one set-up: Looking with more eyes into the intricate chemistry of the synthesis and working of heterogeneous catalysts. Chemical Society Reviews, 2010, 39(12): 4718-4730

65. Wachs I E, Routray K. Catalysis science of bulk mixed oxides. ACS Catalysis, 2012, 2(6): 1235-1246

66. Wachs I E. Recent conceptual advances in the catalysis science of mixed metal oxide catalytic materials. Catalysis Today, 2005, 100(1): 79-94

67. Tran L, Bañares M A, Rallo R. Modelling the Toxicity of Nanoparticles: Springer International Publishing, 2017

68. Guerrero-Pérez M O, Bañares M A. From conventional in situ to operando studies in Raman spectroscopy. Catalysis Today, 2006, 113(1-2): 48-57

69. Bañares M A, Khatib S J. Structure-activity relationships in alumina-supported molybdena-vanadia catalysts for propane oxidative dehydrogenation. Catalysis Today, 2004, 96(4): 251-257

70. Martínez-Huerta M V, Deo G, Fierro J L G, Bañares M A. Operando Raman-GC study on the structure-activity relationships in $\mathrm{V} 5+/ \mathrm{CeO} 2$ catalyst for ethane oxidative dehydrogenation: The formation of CeVO4. Journal of Physical Chemistry C, 2008, 112(30): 11441-11447

71. Banares M A, Dauphin L, Calvoperez V, Fehlner T P, Wolf E E. Activity and characterization of self-supported model catalysts derived from cobalt-based clusters of clusters: hydrogenation of 1,3-Butadiene. Journal of Catalysis, 1995, 152(2): 396-409 
72. Bañares M A, Dauphin L, Lei X, Cen W, Shang M, Wolf E E, Fehlner T P. Effect of precursor core structure on the hydrogenation of 1,3-butadiene catalyzed by clusterderived model catalysts. Chemistry of Materials, 1995, 7(3): 553-561

73. Bañares M, Patil A N, Fehlner T P, Wolf E E. Novel cluster-derived catalysts for the selective hydrogenation of crotonaldehyde. Catalysis Letters, 1995, 34(3-4): 251-258

74. Deutschmann O, Schwiedemoch R, Maier L I, Chatterjee D. Natural gas conversion in monolithic catalysts: interaction of chemical reactions and transport phenomena. In: Iglesia E, Spivey J J, Fleisch T H, (eds). Studies in Surface Science and Catalysis: Elsevier, 2001, 251-258

75. Meunier F, Reid D, Goguet A, Shekhtman S, Hardacre C, Burch R, Deng W, Flytzanistephanopoulos M. Quantitative analysis of the reactivity of formate species seen by DRIFTS over a Au/Ce(La)O2 water-gas shift catalyst: First unambiguous evidence of the minority role of formates as reaction intermediates. Journal of Catalysis, 2007, 247(2): 277-287

76. Rivallan M, Seguin E, Thomas S, Lepage M, Takagi N, Hirata H, Thibault-Starzyk F. Platinum Sintering on H-ZSM-5 Followed by Chemometrics of $\mathrm{CO}$ Adsorption and 2D Pressure-Jump IR Spectroscopy of Adsorbed Species. Angewandte Chemie International Edition, 2010, 49(4): 785-789

77. Bare S R, Ressler T. Characterization of catalysts in reactive atmospheres by Xray absorption spectroscopy. In: Gates B, Knoezinger H, Jentoft F, (eds). Advances in Catalysis: Academic Press, 2009, 339-465

78. $\quad$ Doronkin D E, Lichtenberg H, Grunwaldt J-D. Cell designs for in situ and operando studies. In: Iwasawa Y, Asakura K, Tada M, (eds). XAFS techniques for catalysts, nanomaterials, and surfaces techniques for catalysts, nanomaterials, and surfaces. Cham: Springer International Publishing, 2017, 75-89

79. Carías-Henriquez A, Pietrzyk S, Dujardin C. Modelling and optimization of IR cell devoted to in situ and operando characterization of catalysts. Catalysis Today, 2013, 205(Supplement C): 134-140

80. Burcham L J, Badlani M, Wachs I E. The origin of the ligand effect in metal oxide catalysts: novel fixed-bed in situ infrared and kinetic studies during methanol oxidation. Journal of Catalysis, 2001, 203(1): 104-121

81. Rasmussen S B, Perez-Ferreras S, Bañares M A, Bazin P, Daturi M. Does pelletizing catalysts influence the efficiency number of activity measurements? Spectrochemical engineering considerations for an accurate operando study. ACS Catalysis, 2012, 3(1): 86-94

82. Lisi L, Pirone R, Russo G, Stanzione V. Cu-ZSM5 based monolith reactors for NO decomposition. Chemical Engineering Journal, 2009, 154(1): 341-347

83. Gibson E K, Zandbergen M W, Jacques S D M, Biao C, Cernik R J, O’Brien M G, Di Michiel M, Weckhuysen B M, Beale A M. Noninvasive spatiotemporal profiling of the processes of impregnation and drying within mMo/Al2O3 catalyst bodies by a combination of X-ray absorption tomography and diagonal offset Raman spectroscopy. ACS Catalysis, 2013, 3(3): 339-347

84. $\quad$ Ferri D, Elsener M, Kröcher O. Methane oxidation over a honeycomb Pd-only three-way catalyst under static and periodic operation. Applied Catalysis B: Environmental, 2018, 220: 67-77

85. Malpartida I, Marie O, Bazin P, Daturi M, Jeandel X. An operando IR study of the unburnt $\mathrm{HC}$ effect on the activity of a commercial automotive catalyst for NH3-SCR. Applied Catalysis B: Environmental, 2011, 102(1): 190-200

86. Ávila P, Montes M, Miró E E. Monolithic reactors for environmental applications: A review on preparation technologies. Chemical Engineering Journal, 2005, 109(1): 11-36 
87. Chen J, Yang H, Wang N, Ring Z, Dabros T. Mathematical modeling of monolith catalysts and reactors for gas phase reactions. Applied Catalysis A: General, 2008, 345(1): 1-11

88. Grunwaldt J-D, Wagner J B, Dunin-Borkowski R E. Imaging catalysts at work: A hierarchical approach from the macro- to the meso- and nano-scale. ChemCatChem, 2013, 5(1): 62-80

89. Goguet A, Stewart C, Touitou J, Morgan K. In situ spatially resolved techniques for the investigation of packed bed catalytic reactors: current status and future outlook of Spaci-FB Advances in Chemical Engineering, 2017, 50: 131-160

90. Rasmussen S B, Portela R, Bazin P, Ávila P, Bañares M A, Daturi M. Transient operando study on the $\mathrm{NH} 3 / \mathrm{NH} 4+$ interplay in $\mathrm{V}-\mathrm{SCR}$ monolithic catalysts. Applied Catalysis B: Environmental, 2018, 224: 109-115

91. Grunwaldt J D, Kimmerle B, Baiker A, Boye P, Schroer C G, Glatzel P, Borca C $\mathrm{N}$, Beckmann F. Catalysts at work: From integral to spatially resolved X-ray absorption spectroscopy. Catalysis Today, 2009, 145(3-4): 267-278

92. van de Water L G A, Bergwerff J A, Nijhuis T A, de Jong K P, Weckhuysen B M. UV-vis microspectroscopy: Probing the initial stages of supported metal oxide catalyst preparation. Journal of the American Chemical Society, 2005, 127(14): 5024-5025

93. Fait M J G, Abdallah R, Linke D, Kondratenko E V, Rodemerck U. A novel multichannel reactor system combined with operando UV/vis diffuse reflectance spectroscopy: Proof of principle. Catalysis Today, 2009, 142(3-4): 196-201

94. García-Casado M, Prieto J, Vico-Ruiz E, Lozano-Diz E, Goberna-Selma C, Bañares M A. High-throughput operando Raman-quadrupole mass spectrometer (QMS) system to screen catalytic systems. Applied Spectroscopy, 2014, 68(1): 69-78

95. Zandbergen M W, Jacques S D M, Weckhuysen B M, Beale A M. Chemical probing within catalyst bodies by diagonal offset Raman spectroscopy. Angewandte Chemie International Edition, 2012, 51(4): 957-960

96. van Schrojenstein Lantman E M, Deckert-Gaudig T, Mank A J G, Deckert V, Weckhuysen B M. Catalytic processes monitored at the nanoscale with tip-enhanced Raman spectroscopy. Nat Nanotechnology, 2012, 7(9): 583-586

97. Li G, Hu D, Xia G, White J M, Zhang C. High throughput operando studies using Fourier transform infrared imaging and Raman spectroscopy. Review of Scientific Instruments, 2008, 79(7): 074101

98. Grunwaldt J-D, Schroer C G. Hard and soft X-ray microscopy and tomography in catalysis: bridging the different time and length scales. Chemical Society Reviews, 2010, 39(12): 4741-4753

99. Jacques S D, Di Michiel M, Kimber S A, Yang X, Cernik R J, Beale A M, Billinge S J. Pair distribution function computed tomography. Nature Communications, 2013, 4: 2536

100. Beale A M, Jacques S D M, Gibson E K, Di Michiel M. Progress towards five dimensional diffraction imaging of functional materials under process conditions. Coordination Chemistry Reviews, 2014, 277-278(0): 208-223

101. Vila F D, Rehr J J, Kelly S D, Bare S R. Operando effects on the structure and dynamics of PtnSnm/Y-Al2O3 from ab initio molecular dynamics and X-ray absorption spectra. The Journal of Physical Chemistry C, 2013, 117(24): 12446-12457

102. O'Brien M G, Jacques S D M, Di Michiel M, Barnes P, Weckhuysen B M, Beale A M. Active phase evolution in single $\mathrm{Ni} / \mathrm{Al} 2 \mathrm{O} 3$ methanation catalyst bodies studied in real time using combined [small mu ]-XRD-CT and [small mu ]-absorption-CT. Chemical Science, 2012, 3(2): 509-523

103. Senecal P, Jacques S D M, Di Michiel M, Kimber S A J, Vamvakeros A, Odarchenko Y, Lezcano-Gonzalez I, Paterson J, Ferguson E, Beale A M. Real-time 
scattering-contrast imaging of a supported cobalt-based catalyst body during activation and Fischer-Tropsch synthesis revealing spatial dependence of particle size and phase on catalytic properties. ACS Catalysis, 2017, 7(4): 2284-2293

104. Ngo C, Dzara M J, Shulda S, Pylypenko S. Spectroscopy and microscopy for characterization of fuel cell catalysts. Electrocatalysts for Low Temperature Fuel Cells: Wiley-VCH Verlag GmbH \& Co. KGaA, 2017, 443-466

105. Zhang C, Gustafson J, Merte L R, Evertsson J, Norén K, Carlson S, Svensson $\mathrm{H}$, Carlsson P-A. An in situ sample environment reaction cell for spatially resolved $\mathrm{x}$-ray absorption spectroscopy studies of powders and small structured reactors. Review of Scientific Instruments, 2015, 86(3): 033112

106. Alrwashdeh S S, Manke I, Markötter H, Klages M, Göbel M, Haußmann J, Scholta J, Banhart J. In operando quantification of three-dimensional water distribution in nanoporous carbon-based layers in polymer electrolyte membrane fuel cells. ACS Nano, 2017, 11(6): 5944-5949

107. Yang Y, Risse S, Mei S, Jafta C J, Lu Y, Stöcklein C, Kardjilov N, Manke I, Gong J, Kochovski Z, Ballauff M. Binder-free carbon monolith cathode material for operando investigation of high performance lithium-sulfur batteries with X-ray radiography. Energy Storage Materials, 2017, 9: 96-104

108. Sezen H, Rockett A A, Suzer S. XPS Investigation of a CdS-Based Photoresistor under Working Conditions: Operando-XPS. Analytical Chemistry, 2012, 84(6): 2990-2994

109. Polcari D, Dauphin-Ducharme P, Mauzeroll J. Scanning electrochemical microscopy: a comprehensive review of experimental parameters from 1989 to 2015. Chemical Reviews, 2016, 116(22): 13234-13278

110. Li F, Ciani I, Bertoncello P, Unwin P R, Zhao J, Bradbury C R, Fermin D J. Scanning electrochemical microscopy of redox-mediated hydrogen evolution catalyzed by two-dimensional assemblies of palladium nanoparticles. The Journal of Physical Chemistry C, 2008, 112(26): 9686-9694

111. Sa J, Fernandes D L A, Aiouache F, Goguet A, Hardacre C, Lundie D, Naeem W, Partridge W P, Stere C. SpaciMS: spatial and temporal operando resolution of reactions within catalytic monoliths. Analyst, 2010, 135(9): 2260-2272

112. Bosco M, Vogel F. Optically accessible channel reactor for the kinetic investigation of hydrocarbon reforming reactions. Catalysis Today, 2006, 116(3): 348-353

113. Horn R, Williams K A, Degenstein N J, Bitsch-Larsen A, Dalle Nogare D, Tupy $S A$, Schmidt L D. Methane catalytic partial oxidation on autothermal $\mathrm{Rh}$ and $\mathrm{Pt}$ foam catalysts: Oxidation and reforming zones, transport effects, and approach to thermodynamic equilibrium. Journal of Catalysis, 2007, 249(2): 380-393

114. Luo J Y, Hou X, Wijayakoon P, Schmieg S J, Li W, Epling W S. Spatially resolving SCR reactions over a Fe/zeolite catalyst. Applied Catalysis B: Environmental, 2011, 102(1-2): 110-119

115. Kopyscinski J, Schildhauer T J, Vogel F, Biollaz S M A, Wokaun A. Applying spatially resolved concentration and temperature measurements in a catalytic plate reactor for the kinetic study of CO methanation. Journal of Catalysis, 2010, 271(2): 262-279

116. Korup O, Mavlyankariev S, Geske M, Goldsmith C F, Horn R. Measurement and analysis of spatial reactor profiles in high temperature catalysis research. Chemical Engineering and Processing: Process Intensification, 2011, 50(10): 998-1009

117. Geske M, Korup O, Horn R. Resolving kinetics and dynamics of a catalytic reaction inside a fixed bed reactor by combined kinetic and spectroscopic profiling. Catalysis Science \& Technology, 2013, 3(1): 169-175 
118. Gladden L F, Mantle M D, Sederman A J. Magnetic Resonance Imaging of Catalysts and Catalytic Processes. In: Gates B C, Knzinger H, (eds). Advances in Catalysis: Academic Press, 2006, 1-75

119. Lysova A A, Koptyug I V. Magnetic resonance imaging methods for in situ studies in heterogeneous catalysis. Chemical Society Reviews, 2010, 39(12): 4585-4601

120. Barskiy D A, Coffey A M, Nikolaou P, Mikhaylov D M, Goodson B M, Branca R T, Lu G J, Shapiro M G, Telkki V-V, Zhivonitko V V, Koptyug I V, Salnikov O G, Kovtunov K V, Bukhtiyarov V I, Rosen M S, Barlow M J, Safavi S, Hall I P, Schröder L, Chekmenev E Y. NMR hyperpolarization techniques of gases. Chemistry - A European Journal, 2017, 23(4): 725-751

121. Kovtunov K V, Barskiy D A, Shchepin R V, Coffey A M, Waddell K W, Koptyug I $\mathrm{V}$, Chekmenev $\mathrm{E} Y$. Demonstration of heterogeneous parahydrogen induced polarization using hyperpolarized agent migration from dissolved $\mathrm{Rh}(\mathrm{I})$ complex to gas phase. Analytical Chemistry, 2014, 86(13): 6192-6196

122. Telkki V V, Zhivonitko V V, Selent A, Scotti G, Leppaniemi J, Franssila S, Koptyug I V. Lab-on-a-chip reactor imaging with unprecedented chemical resolution by Hadamard-encoded remote detection NMR. Angew Chem Int Ed Engl, 2014, 53(42): 11289-11293

123. Gladden L F, Buckley C, Chow P S, Davidson J F, Mantle M D, Sederman A J. 'Looking into' chemical products and processes. Current Applied Physics, 2004, 4(2): 9397

124. Gladden L F, Mantle M D, Sederman A J. Magnetic resonance imaging of catalysts and catalytic processes. Advances in Catalysis, 2006, 50: 1-75

125. Cattaneo A S, Villa D C, Angioni S, Ferrara C, Melzi R, Quartarone E, Mustarelli P. Operando electrochemical NMR microscopy of polymer fuel cells. Energy \& Environmental Science, 2015, 8(8): 2383-2388

126. Britton M M, Sederman A J, Taylor A F, Scott S K, Gladden L F. Magnetic resonance imaging of flow-distributed oscillations. The Journal of Physical Chemistry $A$, 2005, 109(37): 8306-8313

127. Ulpts J, Dreher W, Kiewidt L, Schubert M, Thöming J. In situ analysis of gas phase reaction processes within monolithic catalyst supports by applying NMR imaging methods. Catalysis Today, 2016, 273: 91-98

128. Ulpts J, Kiewidt L, Dreher W, Thöming J. 3D characterization of gas phase reactors with regularly and irregularly structured monolithic catalysts by NMR imaging and modeling. Catalysis Today, 2017, In press: https://doi.org/10.1016/i.cattod.2017.1005.1009cl

129. Zheng Q, Russo-Abegao F J, J. S A, F. G L. Operando determination of the liquid-solid mass transfer coefficient during 1-octene hydrogenation. Chemical Engineering Science, 2017, 171: 614-624

130. Li H, Rivallan M, Thibault-Starzyk F, Travert A, Meunier F C. Effective bulk and surface temperatures of the catalyst bed of FT-IR cells used for in situ and operando studies. Physical Chemistry Chemical Physics, 2013, 15(19): 7321-7327

131. Kellow J C, Wolf E E. Infrared thermography and FTIR studies of catalyst preparation effects on surface reaction dynamics during $\mathrm{CO}$ and ethylene oxidation on $\mathrm{Rh} / \mathrm{SiO} 2$ catalysts. Chemical Engineering Science, 1990, 45(8): 2597-2602

132. Kellow J, Wolf E E. In-situ IR thermography studies of reaction dynamics during CO oxidation on Rh-SiO2 catalysts. Catalysis Today, 1991, 9(1): 47-51

133. Kellow J C, Wolf E E. Propagation of oscillations during ethylene oxidation on a $\mathrm{Rh} / \mathrm{SiO} 2$ catalyst. AIChE Journal, 1991, 37(12): 1844-1848 
134. Koptyug I V, Khomichev A V, Lysova A A, Sagdeev R Z. Spatially resolved NMR thermometry of an operating fixed-bed catalytic reactor. Journal of the American Chemical Society, 2008, 130(32): 10452-10453

135. Lysova A A, Kulikov A V, Parmon V N, Sagdeev R Z, Koptyug I V. Quantitative temperature mapping within an operating catalyst by spatially resolved 27Al NMR. Chemical Communications, 2012, 48(46): 5763-5765

136. Kimmerle B, Grunwaldt J-D, Baiker A, Glatzel P, Boye P, Stephan S, Schroer C G. Visualizing a catalyst at work during the ignition of the catalytic partial oxidation of methane. The Journal of Physical Chemistry C, 2009, 113(8): 3037-3040

137. Cao E, Firth S, McMillan P F, Gavriilidis A. Application of microfabricated reactors for operando Raman studies of catalytic oxidation of methanol to formaldehyde on silver. Catalysis Today, 2007, 126(1-2): 119-126

138. Gänzler A M, Casapu M, Boubnov A, Müller O, Conrad S, Lichtenberg H, Frahm R, Grunwaldt J-D. Operando spatially and time-resolved X-ray absorption spectroscopy and infrared thermography during oscillatory $\mathrm{CO}$ oxidation. Journal of Catalysis, 2015, 328: 216-224

139. Hannemann S, Grunwaldt J-D, van Vegten N, Baiker A, Boye P, Schroer C G. Distinct spatial changes of the catalyst structure inside a fixed-bed microreactor during the partial oxidation of methane over Rh/Al2O3. Catalysis Today, 2007, 126(1-2): 54-63

140. Grunwaldt J-D, Baiker A. Axial variation of the oxidation state of Pt-Rh/Al2O3 during partial methane oxidation in a fixed-bed reactor: An in situ X-ray absorption spectroscopy study. Catalysis Letters, 2005, 99(1): 5-12

141. Fletcher P D I, Haswell S J, Zhang X. Monitoring of chemical reactions within microreactors using an inverted Raman microscopic spectrometer. Electrophoresis, 2003, 24(18): 3239-3245 